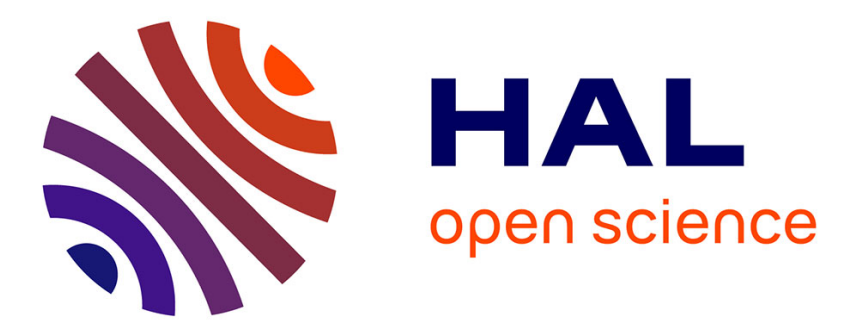

\title{
Fast iterative boundary element methods for high-frequency scattering problems in 3D elastodynamics
}

Stéphanie Chaillat, Marion Darbas, Frédérique Le Louër

\section{To cite this version:}

Stéphanie Chaillat, Marion Darbas, Frédérique Le Louër. Fast iterative boundary element methods for high-frequency scattering problems in 3D elastodynamics. Journal of Computational Physics, 2017, 341, pp.429 - 446. 10.1016/j.jcp.2017.04.020 . hal-01523020

\author{
HAL Id: hal-01523020 \\ https://hal.science/hal-01523020
}

Submitted on 15 May 2017

HAL is a multi-disciplinary open access archive for the deposit and dissemination of scientific research documents, whether they are published or not. The documents may come from teaching and research institutions in France or abroad, or from public or private research centers.
L'archive ouverte pluridisciplinaire HAL, est destinée au dépôt et à la diffusion de documents scientifiques de niveau recherche, publiés ou non, émanant des établissements d'enseignement et de recherche français ou étrangers, des laboratoires publics ou privés. 


\title{
Fast iterative boundary element methods for high-frequency scattering problems in 3D elastodynamics
}

\author{
Stéphanie Chaillat ${ }^{\mathrm{a}, *}$, Marion Darbas ${ }^{\mathrm{b}}$, Frédérique Le Louër $^{\mathrm{c}}$ \\ ${ }^{a}$ Laboratoire POEMS (CNRS-INRIA-ENSTA), Université Paris-Saclay, \\ ENSTA-UMA, 828 Bd des Maréchaux, 91762 Palaiseau Cedex, FRANCE \\ ${ }^{b}$ LAMFA UMR CNRS 7352 \\ Université de Picardie Jules Verne, 33 rue Saint-Leu 80039 Amiens Cedex, FRANCE \\ ${ }^{c} L M A C$ EA 2222 \\ Sorbonne Universités, Université de technologie de Compiègne, \\ CS 60319 - 60203 Compiègne cedex, FRANCE
}

\begin{abstract}
The fast multipole method is an efficient technique to accelerate the solution of large scale 3D scattering problems with boundary integral equations. However, the fast multipole accelerated boundary element method (FM-BEM) is intrinsically based on an iterative solver. It has been shown that the number of iterations can significantly hinder the overall efficiency of the FMBEM. The derivation of robust preconditioners for FM-BEM is now inevitable to increase the size of the problems that can be considered. The main constraint in the context of the FM$\mathrm{BEM}$ is that the complete system is not assembled to reduce computational times and memory requirements. Analytic preconditioners offer a very interesting strategy by improving the spectral properties of the boundary integral equations ahead from the discretization. The main contribution of this paper is to combine an approximate adjoint Dirichlet to Neumann (DtN) map as an analytic preconditioner with a FM-BEM solver to treat Dirichlet exterior scattering problems in 3D elasticity. The approximations of the adjoint DtN map are derived using tools proposed in [40. The resulting boundary integral equations are preconditioned Combined Field Integral Equations (CFIEs). We provide various numerical illustrations of the efficiency of the method for different smooth and non smooth geometries. In particular, the number of iterations is shown to be completely independent of the number of degrees of freedom and of the frequency for convex obstacles.
\end{abstract}

Keywords: Scattering, time-harmonic elastic waves, Boundary Element Method, Fast Multipole Method, Analytical Preconditioner, approximate local DtN map

\section{Introduction}

The paper is concerned with the numerical solution of high-frequency scattering problems of time-harmonic elastic waves by a three-dimensional rigid obstacle. The accurate numerical modeling of highly oscillatory elastic problems is a challenging task. It is a timely research field due to the variety of possible applications (for example seismology, remote sensing or non-destructive testing) [2, 13]. To solve elastodynamic scattering problems in unbounded domains, various numerical methods are used [68. We can mention the Finite Element Method [50, 29, 53, 11] or the Finite Difference Method [65, 47]. Such volume methods are used with nonreflecting boundary conditions [46, 45] such as Absorbing Boundary Conditions (ABCs) 34, 21, 44 or Perfectly Matched Layers (PMLs) [9, 49, 31 to truncate the computational domain. Another possible approach is to use the method of boundary integral equations (BIEs) that enjoys a number of attractive properties (see

\footnotetext{
* Corresponding author

Email addresses: stephanie.chaillat@ensta-paristech.fr (Stéphanie Chaillat) marion.darbas@u-picardie.fr (Marion Darbas), frederique.le-louer@utc.fr (Frédérique Le Louër)
} 
e.g. [12, 51, 56, 55]). The main advantage is to reformulate the exterior boundary value-problem as an integral equation on the boundary of the scatterer. Thus, the dimensionality of the problem is reduced by one. Still, the method has its drawbacks. The discretization matrix of a boundary integral operator is dense. Furthermore, in order to capture the oscillatory phenomenon, one has to fix typically about ten discretization points per wavelength per dimension. The solution of these large and fully-populated complex linear systems is handled by iterative solvers, namely GMRES [69]. On the one hand, the standard Boundary Element Method (BEM) results in high computational costs in terms of computational time $\left(\mathcal{O}\left(N^{2}\right)\right.$ per iteration) and memory requirements $\left(\mathcal{O}\left(N^{2}\right)\right)$, where $N$ denotes the number of degrees of freedom (DOFs) of the BEM model. On the other hand, the spectral properties of the most stable integral equation formulations, the Combined Field Integral Equations (CFIEs), are not particularly well suited for Krylov-subspace iterative solvers such as GMRES. We will see in Section 5 that this main drawback of the CFIEs is exacerbated at high frequencies (see Tables 1 1.5. To decrease the overall cost of the solver, two complementary approaches are considered: (i) fast methods to accelerate the computation of matrix-vector products and (ii) preconditioners to speed up the convergence of the solver.

The Fast Multipole accelerated Boundary Element Method (FM-BEM) is one of the efficient methods used to speed up the evaluation of matrix-vector products when the matrix is obtained by the discretization of an integral operator. The method has been introduced by Rokhlin [64] and then adapted to integral equations for wave propagation problems [36]. The capabilities of the method have been shown in many fields, including in 3D elastodynamics [24]. In practice, the theoretical complexity of the multi-level FMM is $\mathcal{O}(N \log N)$ per iteration both for CPU time and memory requirements. However, the FM-BEM is intrinsically based on an iterative solver and it has been shown that the number of iterations can significantly hinder the overall efficiency of the FM-BEM [25, 26]. The derivation of adequate preconditioners for FM-BEM is now mandatory to increase the size of the problems that can be considered. The main constraint in the context of the FM-BEM is that the complete system is not assembled to reduce computational times and memory requirements.

Traditional algebraic preconditioning approaches such as incomplete LU, SParse Approximative Inverse [19, 20], multi-grid methods [18], nested GMRES algorithm [27] have been applied to electromagnetic or elastodynamic FM-BEMs. However, since algebraic preconditioners use only a small part of the system matrix, they do not contain enough information on the physics of the underlying continuous operator. They show only moderate efficiency for high frequency problems. Analytic preconditioners offer a very interesting alternative. They play the role of regularizing operators in the integral representation of the scattered field and improve the spectral properties of the resulting boundary integral equations ahead from the discretization. This preconditioning strategy based on Calderón's relations has been introduced by Steinbach and Wendland [66] in electromagnetism. Since then, several works have been proposed for constructing Fredholm boundary integral equations of the second kind for both acoustic and electromagnetic scattering problems by closed surfaces (e.g. [5, 6, 157, [58, 1, 15, 16, 39, 14, 63, 30]) or open surfaces (e.g. [32, 33, 4, 17]). Among them, approximations of the DtN map (for Brakhage-Werner type BIEs) or of the adjoint DtN map (for CFIEs) naturally define robust analytical preconditioners when considering Dirichlet boundary value-problems. A pseudo inverse of the principal classical symbol of the single layer boundary integral operator - or equivalently the principal classical symbol of the Neumann trace of the double layer boundary integral operator - is used to approach the DtN map and its adjoint operator [7, 6, 38, in the framework of the On-Surface Radiation Condition (OSRC) methods (e.g. [54, 52, 3]). This is intuitively natural in view of the Calderón's formulas and the compactness of the double layer boundary integral operator. In acoustics, the resulting preconditioner is expressed analytically by a simple square-root of the form $i \sqrt{\Delta_{\Gamma}+\kappa^{2} \mathrm{I}}$. More precisely, a regularization of the square-root, by considering a small complex perturbation of the exterior wavenumber $\kappa$, is considered instead. This allows to model the grazing rays and provides the existence and uniqueness of the solution to the BIEs for any positive real values of the frequency. Furthermore, a complex Padé-approximation of the square-root operator 61] leads to a sparse matrix involving only the mass and rigidity finite element matrices. This makes the implementation of this analytic preconditioner rather easy with a low additional computational 
cost. Furthermore, it can easily be combined with fast methods such as FMM [39] or $\mathcal{H}$-matrices techniques [67] since it simply consists in the application of the preconditioner at each iteration of the iterative solver. This preconditioner has been shown to yield a very fast convergence of GMRES solver and in particular with a number of iterations independent of the frequency and the mesh density.

The main contribution of this paper is to combine such an analytic OSRC-preconditioner with a FM-BEM solver to solve Dirichlet exterior scattering problems in 3D elasticity. The approximations of the adjoint DtN map are derived using strategies proposed in [40, to overcome difficulties inherent to elasticity. For this case, the double layer boundary integral operator and its adjoint are not compact even for sufficiently smooth boundaries. This implies, according to Calderón's identities, that regularizing the standard BIEs via a pseudo inverse of the single layer boundary integral operator is not sufficient to obtain well-conditioned boundary integral equations. The principal part of the double layer boundary integral operator has also to be taken into account in the preconditioner to regularize the single layer integral operator. It is not an easy task to obtain the expressions of the principal parts of each elementary boundary integral operator. To this end, the tangential Günter derivative plays an important role. The well posedness of these preconditioned CFIEs has been proved for smooth boundaries. The approximations of the adjoint DtN map are expressed in terms of surface differential operators, square-root operators and their inverse. To the best of our knowledge, this is the first numerical contribution in this sense in $3 \mathrm{D}$ elastodynamics.

The paper is organized as follows: in Section 2, we introduce the problem setting. We present the Combined Field Integral Equation (CFIE) formulations that are numerically investigated in this paper. Furthermore, we summarize the main lines of the Fast Multipole accelerated Boundary Element Method. In Section 3, we describe the different approximate adjoint DtN maps that we compare and the associated preconditioned CFIEs. Section 4 presents the discretization of the approximate adjoint DtN maps (regularization, local representation of square-root operators). In addition, we give details for an optimal implementation of the CFIEs. In Section 5, we provide various numerical illustrations of the efficiency of the method for different geometries. We address numerical investigation of the eigenvalues of the classical and preconditioned CFIEs when the scatterer is a sphere. The paper ends with some concluding remarks given in Section 6 .

\section{Solution of the Navier exterior problem with boundary integral equations}

\subsection{The Navier exterior problem}

We consider an obstacle represented by a bounded domain $\Omega^{-}$in $\mathbb{R}^{3}$, with a closed boundary $\Gamma:=\partial \Omega^{-}$of class $\mathscr{C}^{2}$ at least. Let $\Omega^{+}$denote the exterior domain $\mathbb{R}^{3} \backslash \overline{\Omega^{-}}$and $\boldsymbol{n}$ the outer unit normal vector to the boundary $\Gamma$. The Lamé parameters $\mu$ and $\lambda$ and the density $\rho$ are positive constants. The propagation of time-harmonic waves in a three-dimensional isotropic and homogeneous elastic medium is governed by the Navier equation [56, Eq. (12.5) page 55]

$$
\mu \boldsymbol{\Delta} \boldsymbol{u}+(\lambda+\mu) \boldsymbol{\nabla} \operatorname{div} \boldsymbol{u}+\rho \omega^{2} \boldsymbol{u}=0
$$

where $\omega>0$ is the frequency. The field $\boldsymbol{u}$ is decomposed into a longitudinal field $\boldsymbol{u}_{p}$ with vanishing curl and a transverse divergence-free field $\boldsymbol{u}_{s}$ solutions to

$$
\boldsymbol{\Delta} \boldsymbol{u}_{p}+\kappa_{p}^{2} \boldsymbol{u}_{p}=0 \text { and } \operatorname{curl} \operatorname{curl} \boldsymbol{u}_{s}-\kappa_{s}^{2} \boldsymbol{u}_{s}=0
$$

with respective wavenumbers $\kappa_{p}^{2}=\rho \omega^{2}(\lambda+2 \mu)^{-1}$ and $\kappa_{s}^{2}=\rho \omega^{2} \mu^{-1}$. The scattering problem is formulated as follows : Given an incident displacement wave $\boldsymbol{u}^{i n c}$ which is assumed to solve the Navier equation in the absence of any scatterer, find the solution $\boldsymbol{u}$ to the Navier equation (1a) in $\Omega^{+}$which satisfies the Dirichlet boundary condition

$$
\boldsymbol{u}=-\boldsymbol{u}^{i n c} \text { on } \Gamma
$$


In addition the behavior of the scattered displacement field $\boldsymbol{u}$ at infinity is described by the Kupradze radiation conditions [56, Eqs (2.6)-(2.9) page 126]

$$
\lim _{r \rightarrow \infty} r\left(\frac{\partial \boldsymbol{u}_{p}}{\partial r}-i \kappa_{p} \boldsymbol{u}_{p}\right)=0, \quad \lim _{r \rightarrow \infty} r\left(\frac{\partial \boldsymbol{u}_{s}}{\partial r}-i \kappa_{s} \boldsymbol{u}_{s}\right)=0, \quad r=|\boldsymbol{x}|,
$$

uniformly in all directions.

We denote by $H_{l o c}^{s}\left(\overline{\Omega^{+}}\right)$and $H^{s}(\Gamma)$ the standard (local in the case of the exterior domain) complex valued, Hilbert-Sobolev spaces of order $s \in \mathbb{R}$ defined on $\overline{\Omega^{+}}$and $\Gamma$ respectively (with the convention $\left.H^{0}=L^{2}\right)$. Spaces of vector functions will be denoted by boldface letters, thus $\boldsymbol{H}^{s}=\left(H^{s}\right)^{3}$. We set $\Delta^{*} \boldsymbol{u}:=\mu \boldsymbol{\Delta} \boldsymbol{u}+(\lambda+\mu) \boldsymbol{\nabla} \operatorname{div} \boldsymbol{u}$. The radiating solution to 1a-1b-1c belongs to the space

$$
\boldsymbol{H}_{+}^{1}\left(\Delta^{*}\right):=\boldsymbol{H}_{l o c}^{1}\left(\Omega^{+}, \Delta^{*}\right):=\left\{\boldsymbol{u} \in \boldsymbol{H}_{l o c}^{1}\left(\overline{\Omega^{+}}\right): \Delta^{*} \boldsymbol{u} \in \boldsymbol{L}_{l o c}^{2}\left(\overline{\Omega^{+}}\right)\right\} .
$$

For existence and uniqueness results, we refer to Kupradze [55, 56].

\subsection{Boundary Integral Equations}

The first main difficulty arising in the numerical solution to the exterior boundary valueproblem $1 \mathrm{a}$ - $-(1 \mathrm{~b})-(1 \mathrm{c})$ is related to the unboundedness of the computational domain $\Omega^{+}$. Integral equation based methods are one of the possible tools to overcome this issue. The approach is based on the potential theory [37]. The Neumann trace, defined by $\boldsymbol{t}_{\mid \Gamma}:=\boldsymbol{T} \boldsymbol{u}$, is given by the traction operator

$$
\boldsymbol{T}=2 \mu \frac{\partial}{\partial \boldsymbol{n}}+\lambda \boldsymbol{n} \operatorname{div}+\mu \boldsymbol{n} \times \mathbf{c u r l} .
$$

We recall that we have $\boldsymbol{u}_{\mid \Gamma} \in \boldsymbol{H}^{\frac{1}{2}}(\Gamma)$ and $\boldsymbol{t}_{\mid \Gamma} \in \boldsymbol{H}^{-\frac{1}{2}}(\Gamma)$ for any $\boldsymbol{u} \in \boldsymbol{H}_{+}^{1}\left(\Delta^{*}\right)$. In the remaining of the paper, we will use the tangential Günter derivative $\mathcal{M}$ defined by [56, Eq. (1.14) page 282]

$$
\mathcal{M}=\frac{\partial}{\partial \boldsymbol{n}}-\boldsymbol{n} \operatorname{div}+\boldsymbol{n} \times \operatorname{curl} .
$$

We also use the surface differential operators: The tangential gradient $\nabla_{\Gamma}$, the surface divergence $\operatorname{div}_{\Gamma}$, the surface scalar curl $\operatorname{curl}_{\Gamma}$, the tangential vector $\operatorname{curl} \operatorname{curl}_{\Gamma}$, the scalar Laplace-Beltrami operator $\Delta_{\Gamma}$ and the vector Laplace-Beltrami operator $\Delta_{\Gamma}$. For their definitions we refer to 62, pages 68-75]. The tangential Günter derivative $\mathcal{M}$ is a surface derivative that is rewritten

$$
\boldsymbol{M} \boldsymbol{u}_{\mid \Gamma}=\left(\left[\boldsymbol{\nabla}_{\Gamma} \boldsymbol{u}_{\mid \Gamma}\right]-\left(\operatorname{div}_{\Gamma} \boldsymbol{u}_{\mid \Gamma}\right) \mathrm{I}_{3}\right) \boldsymbol{n},
$$

where $\left[\boldsymbol{\nabla}_{\Gamma} \boldsymbol{v}\right]$ is the matrix whose the $j$-th column is the tangential gradient of the $j$-th component of $\boldsymbol{v}$. For any real-valued constant $\alpha$, we introduce the modified Neumann trace $\boldsymbol{t}_{\alpha} \in \boldsymbol{H}^{-\frac{1}{2}}(\Gamma)$ defined by

$$
\boldsymbol{t}_{\alpha}=\boldsymbol{t}_{\mid \Gamma}-\alpha \mathcal{M} \boldsymbol{u}_{\mid \Gamma}
$$

For any positive real number $\kappa$, let

$$
G(\kappa, \boldsymbol{x}-\boldsymbol{y})=\frac{e^{i \kappa|\boldsymbol{x}-\boldsymbol{y}|}}{4 \pi|\boldsymbol{x}-\boldsymbol{y}|}
$$

be the fundamental solution of the Helmholtz equation $\Delta v+\kappa^{2} v=0$. Then, the fundamental solution of the Navier equation is written

$$
\Phi(\boldsymbol{x}, \boldsymbol{y})=\frac{1}{\rho \omega^{2}}\left(\operatorname{curl}_{\operatorname{curl}}\left\{G\left(\kappa_{s}, \boldsymbol{x}-\boldsymbol{y}\right) \mathrm{I}_{3}\right\}-\boldsymbol{\nabla}_{\boldsymbol{x}} \operatorname{div}_{\boldsymbol{x}}\left\{G\left(\kappa_{p}, \boldsymbol{x}-\boldsymbol{y}\right) \mathrm{I}_{3}\right\}\right) .
$$


It is a 3 -by-3 matrix-valued function and we have $\Phi(\boldsymbol{x}, \boldsymbol{y})=\Phi(\boldsymbol{x}, \boldsymbol{y})^{\top}=\Phi(\boldsymbol{y}, \boldsymbol{x})$. The single- and double-layer potential operators are defined by

$$
\mathcal{S} \boldsymbol{\varphi}=\int_{\Gamma} \Phi(\cdot, \boldsymbol{y}) \boldsymbol{\varphi}(\boldsymbol{y}) d s(\boldsymbol{y}) \quad \text { and } \quad \mathcal{D}_{\alpha} \boldsymbol{\psi}=\int_{\Gamma}\left[\left(\boldsymbol{T}_{\boldsymbol{y}}-\alpha \mathcal{M}\right) \Phi(\cdot, \boldsymbol{y})\right]^{\top} \boldsymbol{\psi}(\boldsymbol{y}) d s(\boldsymbol{y}),
$$

where $\boldsymbol{T}_{\boldsymbol{y}}=\boldsymbol{T}\left(\boldsymbol{n}(\boldsymbol{y}), \partial_{\boldsymbol{y}}\right)$ and $\boldsymbol{T}_{\boldsymbol{y}} \Phi(\boldsymbol{x}, \boldsymbol{y})$ is the tensor obtained by applying the traction operator $\boldsymbol{T}_{\boldsymbol{y}}$ to each column of $\Phi(\boldsymbol{x}, \boldsymbol{y})$.

The traces of the single- and double-layer potentials are given by applying the exterior Dirichlet and Neumann-type trace to $\mathcal{S}$ and $\mathcal{D}_{\alpha}$ such that we have [40]

$$
\begin{array}{r}
(\mathcal{S} \boldsymbol{\varphi})_{\mid \Gamma}=S \boldsymbol{\varphi}, \quad((\boldsymbol{T}-\alpha \mathcal{M}) \mathcal{S} \boldsymbol{\varphi})_{\mid \Gamma}=-\frac{1}{2} \boldsymbol{\varphi}+D_{\alpha}^{\prime} \boldsymbol{\varphi} \\
\left(\mathcal{D}_{\alpha} \boldsymbol{\psi}\right)_{\mid \Gamma}=\frac{1}{2} \boldsymbol{\psi}+D_{\alpha} \boldsymbol{\psi}, \quad \text { and }\left((\boldsymbol{T}-\alpha \mathcal{M}) \mathcal{D}_{\alpha} \boldsymbol{\psi}\right)_{\mid \Gamma}=N_{\alpha} \boldsymbol{\psi}
\end{array}
$$

where I is the identity operator. Given vector densities $\boldsymbol{\varphi}$ and $\boldsymbol{\psi}$, the boundary integral operators $S, D_{\alpha}, D_{\alpha}^{\prime}$ and $N_{\alpha}$ are defined, for $\boldsymbol{x} \in \Gamma$, by

$$
\begin{aligned}
S \boldsymbol{\varphi}(\boldsymbol{x}) & =\int_{\Gamma} \Phi(\boldsymbol{x}, \boldsymbol{y}) \boldsymbol{\varphi}(\boldsymbol{y}) d s(\boldsymbol{y}) \\
D_{\alpha} \boldsymbol{\psi}(\boldsymbol{x}) & =\int_{\Gamma}\left[\left(\boldsymbol{T}_{\boldsymbol{y}}-\alpha \mathcal{M}\right) \Phi(\boldsymbol{x}, \boldsymbol{y})\right]^{\top} \boldsymbol{\psi}(\boldsymbol{y}) d s(\boldsymbol{y}) \\
D_{\alpha}^{\prime} \boldsymbol{\varphi}(\boldsymbol{x}) & =\int_{\Gamma}\left(\boldsymbol{T}_{\boldsymbol{x}}-\alpha \mathcal{M}\right)\{\Phi(\boldsymbol{x}, \boldsymbol{y}) \boldsymbol{\varphi}(\boldsymbol{y})\} d s(\boldsymbol{y}) \\
N_{\alpha} \boldsymbol{\psi}(\boldsymbol{x}) & =\int_{\Gamma}\left(\boldsymbol{T}_{\boldsymbol{x}}-\alpha \mathcal{M}\right)\left\{\left[\left(\boldsymbol{T}_{\boldsymbol{y}}-\alpha \mathcal{M}\right) \Phi(\boldsymbol{x}, \boldsymbol{y})\right]^{\top} \boldsymbol{\psi}(\boldsymbol{y})\right\} d s(\boldsymbol{y})
\end{aligned}
$$

The operator $S$ is a pseudo-differential operator of order -1, i.e it is bounded from $\boldsymbol{H}^{-\frac{1}{2}}(\Gamma)$ to $\boldsymbol{H}^{\frac{1}{2}}(\Gamma)$ and compact from $\boldsymbol{H}^{-\frac{1}{2}}(\Gamma)$ to itself. The operators $D_{\alpha}$ and $D_{\alpha}^{\prime}$ are of order 0, i.e. they have a strongly singular kernel and are bounded from $\boldsymbol{H}^{\frac{1}{2}}(\Gamma)$ and $\boldsymbol{H}^{-\frac{1}{2}}(\Gamma)$ to themselves, respectively. The operator $N_{\alpha}$ is of order 1, i.e. it has a hypersingular kernel and is bounded from $\boldsymbol{H}^{\frac{1}{2}}(\Gamma)$ to $\boldsymbol{H}^{-\frac{1}{2}}(\Gamma)$. Noting that the modified potential theory consists in replacing the traction operator $\boldsymbol{T}$ with $\boldsymbol{T}-\alpha \mathcal{M}$, the following relations hold

$$
\begin{gathered}
D_{\alpha}^{\prime}=D^{\prime}-\alpha \mathcal{M} S \\
N_{\alpha}=N-\alpha D^{\prime} \mathcal{M}-\alpha \mathcal{M} D+\alpha^{2} \mathcal{M} S \mathcal{M}
\end{gathered}
$$

where $D^{\prime}:=D_{0}^{\prime}$ and $N:=N_{0}$ are the standard elementary boundary integral operators.

There exists various possible integral equations to obtain the Cauchy data $\left(\boldsymbol{u}_{\mid \Gamma}, \boldsymbol{t}_{\mid \Gamma}\right)$. We consider the direct method based on the following integral representation formula

$$
\boldsymbol{u}=-\mathcal{S}\left(\boldsymbol{t}_{\mid \Gamma}+\boldsymbol{t}_{\mid \Gamma}^{i n c}\right)
$$

with $\boldsymbol{t}_{\mid \Gamma}^{i n c}=\boldsymbol{T} \boldsymbol{u}^{i n c}$. Taking the exterior Dirichlet and Neumann-type traces of the right hand side, we obtain on $\Gamma$

$S\left(\boldsymbol{t}_{\mid \Gamma}+\boldsymbol{t}_{\mid \Gamma}^{i n c}\right)=-\boldsymbol{u}_{\mid \Gamma}=\boldsymbol{u}_{\mid \Gamma}^{i n c} \quad$ and $\quad\left(-\frac{\mathrm{I}}{2}+D_{\alpha}^{\prime}\right)\left(\boldsymbol{t}_{\mid \Gamma}+\boldsymbol{t}_{\mid \Gamma}^{i n c}\right)=-\boldsymbol{t}_{\alpha} \Leftrightarrow\left(\frac{\mathrm{I}}{2}+D_{\alpha}^{\prime}\right)\left(\boldsymbol{t}_{\mid \Gamma}+\boldsymbol{t}_{\mid \Gamma}^{i n c}\right)=\boldsymbol{t}_{\alpha}^{i n c}$.

Combining the previous two equations, we construct the well-known CFIE: find the physical unknown $\boldsymbol{\varphi}=-\left(\boldsymbol{t}_{\mid \Gamma}+\boldsymbol{t}_{\mid \Gamma}^{i n c}\right) \in \boldsymbol{H}^{-\frac{1}{2}}(\Gamma)$ solution to

$$
\left(\frac{\mathrm{I}}{2}+D_{\alpha}^{\prime}+i \eta S\right) \varphi=-\left(\boldsymbol{t}_{\alpha}^{i n c}+i \eta \boldsymbol{u}_{\mid \Gamma}^{i n c}\right), \quad \text { on } \Gamma,
$$


with $\eta$ a non-zero real constant. The integral equation 8 is well-posed for any frequency $\omega$ and any non-zero real parameter $\eta[51,55,56]$. The choice $\alpha=0$ leads to the standard CFIE

$$
\left(\frac{\mathrm{I}}{2}+D^{\prime}+i \eta S\right) \varphi=-\left(\boldsymbol{t}^{i n c}+i \eta \boldsymbol{u}_{\mid \Gamma}^{i n c}\right), \quad \text { on } \Gamma
$$

which is the most stable integral equation and thus it is considered as the reference one in this paper. Hähner and Hsiao [48] have shown that for $\alpha=\alpha_{H H}:=\left(2 \mu^{2}\right) /(\lambda+3 \mu)$, the CFIE (8) (called Hähner-Hsiao CFIE in the sequel) is of the second-kind for any positive real-valued parameter $\eta$.

\subsection{Fast Multipole accelerated Boundary Element Method for $3 D$ elastodynamics}

The main ingredients of the Boundary Element Method are a transposition of the concepts developed for the Finite Element Method. First, the numerical solution of boundary integral equation (8) is based on a discretization of the surface $\Gamma$ into $N_{E}$ boundary elements (three-noded triangles in this work). Each component of the total traction field is interpolated with continuous piecewise-linear shape functions. The $N_{I}$ interpolation points are chosen as the vertices of the mesh. To discretize the boundary integral equation (8) we consider the collocation approach. It consists in enforcing the equation at a finite number of collocation points $\boldsymbol{x}$. The $N_{I}$ traction interpolation nodes also serve as collocation points. This discretization process transforms (8) into a square complex-valued linear system of size $N=3 N_{I}$ of the form

$$
[K]\{\varphi\}=\{f\}
$$

where the $N$-vector $\{\varphi\}$ collects the sought degrees of freedom (DOFs), namely the nodal traction components, while the $N \times N$ matrix of influence coefficients $[K]$ and the $N$-vector $\{f\}$ arise from (8). Setting up the matrix $[K]$ classically requires the computation of all element integrals for each collocation point, thus needing a computational time of order $\mathcal{O}\left(N^{2}\right)$. To lower this $\mathcal{O}\left(N^{2}\right)$ complexity, which is unacceptable for large BEM models, fast BEM solutions techniques such as the Fast Multipole Method (FMM) must be employed.

The goal of the FMM is to speed up the matrix-vector product computation required for each iteration of the iterative solver applied to the BEM-discretized equations. Moreover, the governing BEM matrix is never explicitly formed, which leads to a storage requirement well below the $\mathcal{O}\left(N^{2}\right)$ memory required for holding it. Substantial savings in both CPU time and memory requirements are thus achieved. In general terms, the FMM exploits a reformulation of the fundamental solutions in terms of products of functions of $\boldsymbol{x}$ and of $\boldsymbol{y}$, so that (unlike in the traditional BEM) integrations with respect to $\boldsymbol{y}$ can be reused when the collocation point $\boldsymbol{x}$ is changed. On decomposing the position vector $\boldsymbol{r}=\boldsymbol{y}-\boldsymbol{x}$ into $\boldsymbol{r}=\left(\boldsymbol{y}-\boldsymbol{y}_{0}\right)+\boldsymbol{r}_{\mathbf{0}}-\left(\boldsymbol{x}-\boldsymbol{x}_{0}\right)$, where $\boldsymbol{x}_{0}$ and $\boldsymbol{y}_{0}$ are two poles and $\boldsymbol{r}_{\mathbf{0}}=\boldsymbol{y}_{0}-\boldsymbol{x}_{0}$ (Fig. 1) and invoking a plane wave decomposition, the Helmholtz Green's function is written as [41]:

$$
G(\kappa,|\mathbf{r}|)=\lim _{L \rightarrow+\infty} \int_{\hat{\boldsymbol{s}} \in S} e^{i \kappa \hat{\boldsymbol{s}} \cdot\left(\boldsymbol{y}-\boldsymbol{y}_{0}\right)} \mathcal{G}_{L}\left(\hat{\boldsymbol{s}} ; \boldsymbol{r}_{\mathbf{0}} ; \kappa\right) e^{-i \kappa \hat{\boldsymbol{s}} \cdot\left(\boldsymbol{x}-\boldsymbol{x}_{0}\right)} d \hat{\boldsymbol{s}}
$$

where $S$ is the unit sphere of $\mathbb{R}^{3}$ and the transfer function $\mathcal{G}_{L}\left(\hat{\boldsymbol{s}} ; \boldsymbol{r}_{\mathbf{0}} ; k\right)$ is defined in terms of the Legendre polynomials $P_{p}$ and the spherical Hankel functions of the first $\operatorname{kind} h_{p}^{(1)}$ by:

$$
\mathcal{G}_{L}\left(\hat{\boldsymbol{s}} ; \boldsymbol{r}_{0} ; \kappa\right)=\frac{i \kappa}{16 \pi^{2}} \sum_{0 \leq p \leq L}(2 p+1) i^{p} h_{p}^{(1)}\left(\kappa\left|\boldsymbol{r}_{0}\right|\right) P_{p}\left(\cos \left(\hat{\boldsymbol{s}}, \boldsymbol{r}_{\mathbf{0}}\right)\right)
$$

The elastodynamic fundamental solution (3) is easily seen to admit representations of the form (11) with $\mathcal{G}_{L}$ replaced with suitably-defined (tensor) transfer functions $\mathcal{U}_{L}^{p}$ and $\mathcal{U}_{L}^{s}$ (see [25] for more 


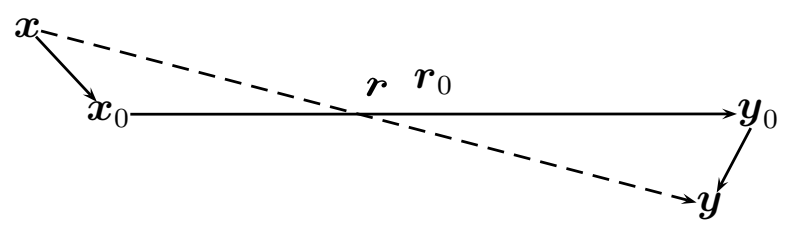

Figure 1: Decomposition of the position vector for the Fast Multipole Method: notations.

details on the expressions and derivation of these transfer functions):

$$
\begin{aligned}
\Phi(\boldsymbol{x}, \boldsymbol{y})=\lim _{L \rightarrow+\infty} \int_{\hat{\boldsymbol{s}} \in S} e^{i \kappa_{p} \hat{\boldsymbol{s}} \cdot\left(\boldsymbol{y}-\boldsymbol{y}_{0}\right)} \mathcal{U}_{L}^{p}\left(\hat{\boldsymbol{s}} ; \boldsymbol{r}_{\mathbf{0}}\right) e^{-i \kappa_{p} \hat{\boldsymbol{s}} \cdot\left(\boldsymbol{x}-\boldsymbol{x}_{0}\right)} d \hat{\boldsymbol{s}} \\
\\
\quad+\lim _{L \rightarrow+\infty} \int_{\hat{\boldsymbol{s}} \in S} e^{i \kappa_{s} \hat{\boldsymbol{s}} \cdot\left(\boldsymbol{y}-\boldsymbol{y}_{0}\right)} \mathcal{U}_{L}^{s}\left(\hat{\boldsymbol{s}} ; \boldsymbol{r}_{\mathbf{0}}\right) e^{-i \kappa_{s} \hat{\boldsymbol{s}} \cdot\left(\boldsymbol{x}-\boldsymbol{x}_{0}\right)} d \hat{\boldsymbol{s}} .
\end{aligned}
$$

It can be shown that expression 13 can be used only for well-separated sets of collocation and integration points clustered around poles $\boldsymbol{x}_{0}$ and $\boldsymbol{y}_{0}$.

In the single-level FMM, a 3D cubic grid of linear spacing $d$ embedding the whole boundary $\Gamma$ is then introduced to drive the computation (see Fig. 22). The FMM basically consists in using decomposition (13), with the poles $\boldsymbol{x}_{0}$ and $\boldsymbol{y}_{0}$ being chosen as the cell centers, whenever $\boldsymbol{x}$ and $\boldsymbol{y}$ belong to non-adjacent cubic cells (i.e. cells that do not share a corner, Fig. 3). The treatment of the fast multipole ("FM") contributions exploits the plane wave decomposition (13) of the fundamental solution, truncated at a finite $L$ and in a manner suggested by its multiplicative form. For instance, the efficient evaluation of the matrix-vector product $S \varphi$ needed in $(8)$ is decomposed into three steps:

1. Evaluation of the multipole moments for each cell $\mathcal{C}_{y}$

$$
\mathcal{R}^{p}\left(\hat{\boldsymbol{s}} ; \mathcal{C}_{y}\right)=\int_{\Gamma} e^{i \kappa_{p} \hat{\boldsymbol{s}} \cdot\left(\boldsymbol{y}-\boldsymbol{y}_{0}\right)} \boldsymbol{\varphi}(\boldsymbol{y}) d s(\boldsymbol{y}) \quad \text { and } \quad \mathcal{R}^{s}\left(\hat{\boldsymbol{s}} ; \mathcal{C}_{y}\right)=\int_{\Gamma} e^{i \kappa_{s} \hat{\boldsymbol{s}} \cdot\left(\boldsymbol{y}-\boldsymbol{y}_{0}\right)} \boldsymbol{\varphi}(\boldsymbol{y}) d s(\boldsymbol{y}) .
$$

2. Application of the truncated tensor transfer functions

$$
\mathcal{L}^{p}\left(\hat{\boldsymbol{s}} ; \mathcal{C}_{x}\right)=\sum_{\mathcal{C}_{y} \notin \mathcal{A}\left(\mathcal{C}_{x}\right)} \mathcal{U}_{L}^{p}\left(\hat{\boldsymbol{s}} ; \boldsymbol{r}_{\mathbf{0}}\right) \mathcal{R}^{p}\left(\hat{\boldsymbol{s}} ; \mathcal{C}_{y}\right) \quad \text { and } \quad \mathcal{L}^{s}\left(\hat{\boldsymbol{s}} ; \mathcal{C}_{x}\right)=\sum_{\mathcal{C}_{y} \notin \mathcal{A}\left(\mathcal{C}_{x}\right)} \mathcal{U}_{L}^{s}\left(\hat{\boldsymbol{s}} ; \boldsymbol{r}_{\mathbf{0}}\right) \mathcal{R}^{s}\left(\hat{\boldsymbol{s}} ; \mathcal{C}_{y}\right) .
$$

3. Numerical evaluation of the integration over the unit sphere with a quadrature rule

$$
(S \boldsymbol{\varphi})^{F M}(\boldsymbol{x}) \approx \sum_{q} w_{q}\left[e^{-i \kappa_{p} \hat{\boldsymbol{s}}_{q} \cdot\left(\boldsymbol{x}-\boldsymbol{x}_{0}\right)} \mathcal{L}^{p}\left(\hat{\boldsymbol{s}}_{q} ; \mathcal{C}_{x}\right)+e^{-i \kappa_{s} \hat{\boldsymbol{s}}_{q} \cdot\left(\boldsymbol{x}-\boldsymbol{x}_{0}\right)} \mathcal{L}^{s}\left(\hat{\boldsymbol{s}}_{q} ; \mathcal{C}_{x}\right)\right] .
$$

Conversely, when $\boldsymbol{x}$ and $\boldsymbol{y}$ belong to adjacent cells, traditional BEM evaluation methods based on (3) are used instead.

To improve further the computational efficiency of the FM-BEM, standard (i.e. non-FMM) calculations must be confined to the smallest possible spatial regions while retaining the advantage of clustering the computation of influence terms into non-adjacent large groups whenever possible. This is achieved by recursively subdividing cubic cells into eight smaller cubic cells. New pairs of non-adjacent smaller cells, to which plane wave expansions are applicable, are thus obtained from the subdivision of pairs of adjacent cells. The cell-subdivision approach is systematized by means of an octree structure of cells. At each level $\ell$, the linear cell size is denoted $d^{\ell}$. The level $\ell=0$, composed of only one cubic cell containing the whole surface $\partial \Omega$, is the tree root. The subdivision process is further repeated until the finest level $\ell=\bar{\ell}$, implicitly defined by a preset subdivision-stopping criterion $\left(d^{\bar{\ell}} \geq d^{\mathrm{min}}\right)$, is reached. Level- $\bar{\ell}$ cells are usually termed leaf cells. This is the essence of the multi-level FMM, whose theoretical complexity is $O(N \log N)$ per 


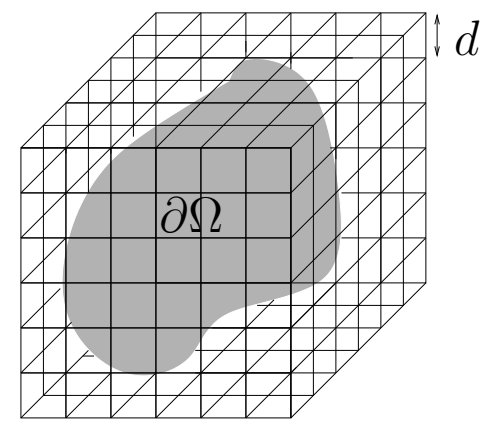

Figure 2: 3D cubic grid embedding the boundary.

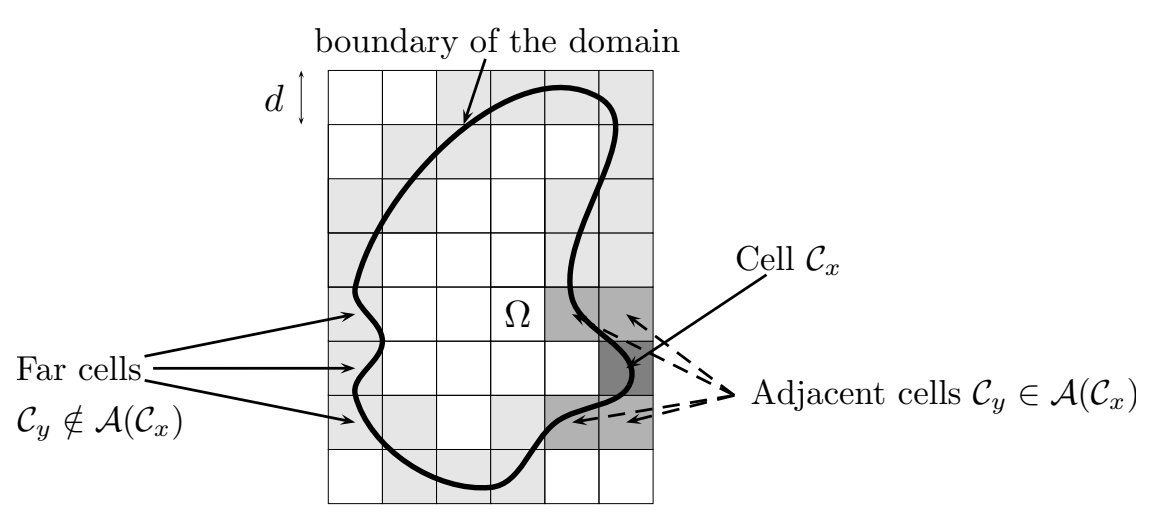

Figure 3: Definition of the adjacent cells.

GMRES iteration both for CPU time and memory (see 25] for further details on the method and its implementation for single-domain elastodynamic problems).

The FM-BEM has been shown to be very efficient to solve elastodynamic problems (see 23] for a review on recent advances). But the main limitation is now the number of iterations required to achieve convergence in GMRES. Since the complete matrix is not explicitly assembled to reduce memory requirements, standard algebraic preconditioners based on the matrix of the near contributions are moderately robust 28]. For these reasons, we consider in the following wellconditioned integral equations based on an approximation of the adjoint Dirichlet-to-Neumann (DtN) map. Such analytical preconditioners have been presented theoretically and numerically in acoustics in [6, 39]. First theoretical investigations in elastodynamics [40] lead to various possible well-conditioned integral equations. We discuss in this work the optimal numerical strategy.

\section{Approximation of the adjoint DtN operator for well-conditioned boundary integral equations}

It is well known that the standard CFIE is not suited for an iterative solution. Even though the Hähner-Hsiao CFIE is more adapted (because of second kind), CFIE (8) does not provide sufficiently good spectral properties at high frequencies (see Section 5). To expect an eigenvalue clustering and hence a fast convergence of GMRES, we need to derive well-conditioned boundary integral equations. The approach we consider is to use an approximation of the adjoint DtN map. The exact exterior Modified Dirichlet-to-Neumann (MDtN) map is defined by

$$
\boldsymbol{\Lambda}_{\alpha}^{\mathrm{ex}}: \boldsymbol{u}_{\mid \Gamma} \in \boldsymbol{H}^{\frac{1}{2}}(\Gamma) \mapsto \boldsymbol{\Lambda}_{\alpha}^{\mathrm{ex}} \boldsymbol{u}_{\mid \Gamma}:=\boldsymbol{t}_{\alpha} \in \boldsymbol{H}^{-\frac{1}{2}}(\Gamma) .
$$


Obviously, the case $\alpha=0$ corresponds to the exact DtN map

$$
\boldsymbol{\Lambda}^{\mathrm{ex}}: \boldsymbol{u}_{\mid \Gamma} \in \boldsymbol{H}^{\frac{1}{2}}(\Gamma) \mapsto \boldsymbol{\Lambda}^{\mathrm{ex}} \boldsymbol{u}_{\mid \Gamma}:=\boldsymbol{t}_{\mid \Gamma} \in \boldsymbol{H}^{-\frac{1}{2}}(\Gamma),
$$

and we have $\boldsymbol{\Lambda}^{\mathrm{ex}}=\boldsymbol{\Lambda}_{\alpha}^{\mathrm{ex}}+\alpha \mathcal{M}$. We find, by taking the exterior Dirichlet trace of the Somigliana integral representation formula of the scattered field, that the exact MDtN operator satisfies on $\Gamma$ 40

$$
\frac{\mathrm{I}}{2}+D_{\alpha}-S \boldsymbol{\Lambda}_{\alpha}^{\mathrm{ex}}=\mathrm{I}
$$

In order to avoid the use of non-physical quantities, we consider the $L^{2}$-adjoint form of the abovewritten boundary integral equation operator

$$
\frac{\mathrm{I}}{2}+D_{\alpha}^{\prime}-\Lambda_{\alpha}^{\mathrm{ex}^{\prime}} S=\mathrm{I}
$$

that is related to the CFIE (8). The adjoint MDtN map $\Lambda_{\alpha}^{\mathrm{ex}^{\prime}}: \boldsymbol{H}^{\frac{1}{2}}(\Gamma) \rightarrow \boldsymbol{H}^{-\frac{1}{2}}(\Gamma)$ satisfies

$$
\boldsymbol{\Lambda}^{\mathrm{ex}^{\prime}}=\boldsymbol{\Lambda}_{\alpha}^{\mathrm{ex}^{\prime}}+\alpha \mathcal{M}
$$

Assume that $\omega$ is not an eigenfrequency of the Navier equation 1 ia $\Omega^{-}$with either the Dirichlet or the Neumann homogeneous boundary condition, the adjoint MDtN map is expressed in terms of boundary integral operators on $\Gamma$ by

$$
\boldsymbol{\Lambda}_{\alpha}^{\mathrm{ex}^{\prime}}=-\left(\frac{1}{2} \mathrm{I}-D_{\alpha}^{\prime}\right) S^{-1}=\left(\frac{1}{2} \mathrm{I}+D_{\alpha}^{\prime}\right)^{-1} N_{\alpha}
$$

In the ideal configuration (14), the solution $\boldsymbol{\varphi}=-\left(\boldsymbol{t}_{\alpha}^{i n c}-\boldsymbol{\Lambda}_{\alpha}^{\mathrm{ex}^{\prime}} \boldsymbol{u}_{\mid \Gamma}^{i n c}\right)$ is obtained directly. However, it is too expensive to consider the exact operator 16 for a numerical purpose. Instead, an approximation $\boldsymbol{\Lambda}_{\alpha}^{\prime}$ of $\boldsymbol{\Lambda}_{\alpha}^{\mathrm{ex}}$, given in terms of surface differential operators, is introduced to construct a preconditioned CFIE : Find $\boldsymbol{\varphi}=-\left(\boldsymbol{t}_{\mid \Gamma}+\boldsymbol{t}_{\mid \Gamma}^{i n c}\right)$ solution to

$$
\left(\frac{\mathrm{I}}{2}+D_{\alpha}^{\prime}-\boldsymbol{\Lambda}_{\alpha}^{\prime} S\right) \boldsymbol{\varphi}=-\left(\boldsymbol{t}_{\alpha}^{i n c}-\boldsymbol{\Lambda}_{\alpha}^{\prime} \boldsymbol{u}_{\mid \Gamma}^{i n c}\right), \quad \text { on } \Gamma
$$

Coming back to the standard case $\alpha=0$, solving equation 17 is equivalent to solving

$$
\left(\frac{\mathrm{I}}{2}+D^{\prime}-\boldsymbol{\Lambda}^{\prime} S\right) \boldsymbol{\varphi}=-\left(\boldsymbol{t}_{\mid \Gamma}^{i n c}-\boldsymbol{\Lambda}^{\prime} \boldsymbol{u}_{\mid \Gamma}^{i n c}\right), \quad \text { on } \Gamma
$$

where the approximate adjoint DtN map is given by the formula $\boldsymbol{\Lambda}^{\prime}=\boldsymbol{\Lambda}_{\alpha}^{\prime}+\alpha \mathcal{M}$. Indeed, we consider the definition (6) of the double-layer boundary integral operator to write

$$
\begin{aligned}
\frac{\mathrm{I}}{2}+D_{\alpha}^{\prime}-\boldsymbol{\Lambda}_{\alpha}^{\prime} S & =\frac{\mathrm{I}}{2}+D^{\prime}-\alpha \mathcal{M} S-\left(\boldsymbol{\Lambda}^{\prime}-\alpha \mathcal{M}\right) S \\
& =\frac{\mathrm{I}}{2}+D^{\prime}-\boldsymbol{\Lambda}^{\prime} S
\end{aligned}
$$

and we also obtain

$$
\boldsymbol{t}_{\alpha}^{i n c}-\boldsymbol{\Lambda}_{\alpha}^{\prime} \boldsymbol{u}_{\mid \Gamma}^{i n c}=\boldsymbol{t}_{\mid \Gamma}^{i n c}-\alpha \boldsymbol{M} \boldsymbol{u}_{\mid \Gamma}^{i n c}-\left(\boldsymbol{\Lambda}^{\prime}-\alpha \boldsymbol{M}\right) \boldsymbol{u}_{\mid \Gamma}^{i n c}=\boldsymbol{t}_{\mid \Gamma}^{i n c}-\boldsymbol{\Lambda}^{\prime} \boldsymbol{u}_{\mid \Gamma}^{i n c} .
$$

The spectral properties of 18 depend on the choice of the approximate adjoint DtN map $\boldsymbol{\Lambda}^{\prime}$. We want to compare several approximations of different orders. In [40, new approximations of the DtN map have been obtained in the spirit of OSRC methods that can be considered when dealing with the adjoint operator. The idea is to consider only the principal part of the exact 
adjoint operator $\Lambda_{\alpha}^{\mathrm{ex}^{\prime}}$ and the judicious decomposition 16 . The approximation is defined by

$$
\left\{\begin{array}{l}
\boldsymbol{\Lambda}_{\alpha}^{\prime}=\left(\frac{\mathrm{I}}{2}+P\left(D_{\alpha}^{\prime}\right)\right)^{-1} P\left(N_{\alpha}\right) \\
\boldsymbol{\Lambda}^{\prime}=\boldsymbol{\Lambda}_{\alpha}^{\prime}+\alpha \mathcal{M}
\end{array}\right.
$$

where the operators $P\left(D_{\alpha}^{\prime}\right)$ and $P\left(N_{\alpha}\right)$ are respectively the principal parts of the boundary integral operators $D_{\alpha}^{\prime}$ and $N_{\alpha}$. We point out that the operator $\boldsymbol{\Lambda}^{\prime}$ is not obtained from the principal symbol of $\Lambda^{\mathrm{ex}^{\prime}}$, but using the formula (15). Thus it depends on $\alpha$ that has to be choosen to reduce the computational cost to obtain $\left(\frac{\mathrm{I}}{2}+P\left(D_{\alpha}^{\prime}\right)\right)^{-1}$.

We provide explicit expressions of the principal parts $P\left(D_{\alpha}^{\prime}\right)$ and $P\left(N_{\alpha}\right)$ using tools proposed in [40]. The operator $P\left(D_{\alpha}^{\prime}\right)$ is decomposed into two terms: $P\left(D_{\alpha}^{\prime}\right)=I_{1}+I_{2}$ with

$$
\begin{aligned}
I_{1}= & \frac{i}{2}\left(\boldsymbol{n}\left(\Delta_{\Gamma}+\kappa_{p}^{2} \mathrm{I}\right)^{-\frac{1}{2}} \operatorname{div}_{\Gamma} \mathrm{I}_{\boldsymbol{t}}-\boldsymbol{\nabla}_{\Gamma}\left(\Delta_{\Gamma}+\kappa_{s}^{2} \mathrm{I}\right)^{-\frac{1}{2}} \boldsymbol{n} \cdot \mathrm{I}_{\boldsymbol{n}}\right) \\
I_{2}=\frac{i(2 \mu-\alpha)}{2 \rho \omega^{2}}( & -\boldsymbol{n}\left(\Delta_{\Gamma}+\kappa_{s}^{2} \mathrm{I}\right)^{\frac{1}{2}} \operatorname{div}_{\Gamma} \mathrm{I}_{\boldsymbol{t}}+\boldsymbol{n} \Delta_{\Gamma}\left(\Delta_{\Gamma}+\kappa_{p}^{2} \mathrm{I}\right)^{-\frac{1}{2}} \operatorname{div}_{\Gamma} \mathrm{I}_{\boldsymbol{t}} \\
& \left.+\boldsymbol{\nabla}_{\Gamma}\left(\Delta_{\Gamma}+\kappa_{p}^{2} \mathrm{I}\right)^{\frac{1}{2}}\left(\boldsymbol{n} \cdot \mathrm{I}_{\boldsymbol{n}}\right)-\boldsymbol{\nabla}_{\Gamma}\left(\Delta_{\Gamma}+\kappa_{s}^{2} \mathrm{I}\right)^{-\frac{1}{2}} \Delta_{\Gamma}\left(\boldsymbol{n} \cdot \mathrm{I}_{\boldsymbol{n}}\right)\right)
\end{aligned}
$$

where $\mathrm{I}_{\boldsymbol{n}}=\boldsymbol{n} \otimes \boldsymbol{n}$ and $\mathrm{I}_{\boldsymbol{t}}=\mathrm{I}-\mathrm{I}_{\boldsymbol{n}}$.

The operator $P\left(N_{\alpha}\right)$ is decomposed into three terms $P\left(N_{\alpha}\right)=J_{1}+J_{2}+J_{3}$ by setting

$$
\begin{aligned}
J_{1}= & \frac{i}{2}\left((\lambda+2 \mu) \kappa_{p}^{2} \boldsymbol{n}\left(\Delta_{\Gamma}+\kappa_{p}^{2} \mathrm{I}\right)^{-\frac{1}{2}} \boldsymbol{n} \cdot \mathrm{I}_{\boldsymbol{n}}+\mu\left(\boldsymbol{\Delta}_{\Gamma}+\kappa_{s}^{2} \mathrm{I}\right)^{-\frac{1}{2}}\left(\kappa_{s}^{2} \mathrm{I}_{\boldsymbol{t}}-\operatorname{curl}_{\Gamma} \operatorname{curl}_{\Gamma}\right)\right) \\
J_{2}= & -i(\alpha-2 \mu)\left(\boldsymbol{\nabla}_{\Gamma}\left(\Delta_{\Gamma}+\kappa_{s}^{2} \mathrm{I}\right)^{-\frac{1}{2}} \operatorname{div}_{\Gamma} \mathrm{I}_{\boldsymbol{t}}+\boldsymbol{n} \Delta_{\Gamma}\left(\Delta_{\Gamma}+\kappa_{p}^{2} \mathrm{I}\right)^{-\frac{1}{2}} \boldsymbol{n} \cdot \mathrm{I}_{\boldsymbol{n}}\right) \\
J_{3}= & \frac{i\left((\alpha-2 \mu)^{2}\right.}{2 \rho \omega^{2}}\left(-\boldsymbol{n}\left(\Delta_{\Gamma}+\kappa_{s}^{2} \mathrm{I}\right)^{\frac{1}{2}} \Delta_{\Gamma}\left(\boldsymbol{n} \cdot \mathrm{I}_{\boldsymbol{n}}\right)+\boldsymbol{n} \Delta_{\Gamma}\left(\Delta_{\Gamma}+\kappa_{p}^{2} \mathrm{I}\right)^{-\frac{1}{2}} \Delta_{\Gamma}\left(\boldsymbol{n} \cdot \mathrm{I}_{\boldsymbol{n}}\right)\right. \\
& \left.-\boldsymbol{\nabla}_{\Gamma}\left(\Delta_{\Gamma}+\kappa_{p}^{2} \mathrm{I}\right)^{\frac{1}{2}} \operatorname{div}_{\Gamma} \mathrm{I}_{\boldsymbol{t}}+\boldsymbol{\nabla}_{\Gamma}\left(\Delta_{\Gamma}+\kappa_{s}^{2} \mathrm{I}\right)^{-\frac{1}{2}} \Delta_{\Gamma} \operatorname{div}_{\Gamma} \mathrm{I}_{\boldsymbol{t}}\right) .
\end{aligned}
$$

Square-root operators of the form $\left(\Delta_{\Gamma}+\kappa_{\gamma}^{2} \mathrm{I}\right)^{1 / 2}$ with $\gamma=s, p$, and their inverse appear in the preconditioner $\boldsymbol{\Lambda}^{\prime}$. The square-root $z^{1 / 2}$ of a complex number $z$ stands for the classical complex square-root with branch-cut along the negative real axis. An artificial singularity of square-root operators occurs in the transition zone from the propagating modes to the evanescent ones. The presence of the singularity does not allow a satisfactory representation of the grazing modes. To model the behavior in the transition zone, we use a regularization [7 by adding a small local damping parameter $\varepsilon_{\gamma}>0$ to the wavenumber $\kappa_{\gamma}$. We set $\kappa_{\gamma, \varepsilon}:=\kappa_{\gamma}+i \varepsilon_{\gamma}$ and we consider square-root operators $\left(\Delta_{\Gamma}+\kappa_{\gamma, \varepsilon}^{2} \mathrm{I}\right)^{1 / 2}$ and their inverse in the preconditioner $\boldsymbol{\Lambda}^{\prime}$. The addition of a local damping is important to obtain the well-posed character of the corresponding preconditioned CFIEs. For existence and uniqueness results, we refer to [40].

From (19), we derive several adjoint DtN approximations.

Low-order adjoint DtN approximation. We retain in $(19)$ the informations associated to the first eigenmode of the scalar and vector Laplacian Beltrami operators only. We obtain the following adjoint DtN approximation (independent of $\alpha$ )

$$
\boldsymbol{\Lambda}^{\prime}:=\boldsymbol{\Lambda}_{\mathrm{LO}}^{\prime}=i\left((\lambda+2 \mu) \kappa_{p} \mathrm{I}_{\boldsymbol{n}}+\mu \kappa_{s} \mathrm{I}_{\boldsymbol{t}}\right) .
$$

More precisely, the operator 22 is contained in the term $J_{1}(21)$ where we have replaced the surface differential operators by their first eigenvalue equal to zero. This approximation of the 
adjoint DtN operator corresponds to the radiation conditions given in [56, Theorem 2.9 page 127] which are equivalent to Kupradze ones (1c). Such an approximation was previously used in the framework of nonreflecting boundary conditions when the artificial boundary is a sphere [45]. The associated preconditioned integral equation is

$$
\left(\frac{\mathrm{I}}{2}+D^{\prime}-\boldsymbol{\Lambda}_{\mathrm{LO}}^{\prime} S\right) \boldsymbol{\varphi}=-\left(\boldsymbol{t}_{\mid \Gamma}^{i n c}-\boldsymbol{\Lambda}_{\mathrm{LO}}^{\prime} \boldsymbol{u}_{\mid \Gamma}^{i n c}\right), \quad \text { on } \Gamma,
$$

is called LO-preconditioned CFIE (LO P-CFIE) in the remaining of the paper. This preconditioner is easy to implement and this is its main advantage. By construction, this approximation provides a good clustering of the first eigenvalues (associated with propagating modes) only. We will show that we need high-order approximations to also cluster grazing and evanescent modes, and then to obtain a more efficient preconditioner (see Section 5 for numerical evidences).

High-order adjoint DtN approximations. The approximation (19) depends on the parameter $\alpha$ and thus provides an infinity of high-order approximate adjoint DtN maps. Among all the possible values of $\alpha$, we choose $\alpha=\alpha_{H H}$ for which the operator $D_{\alpha}$ and its adjoint are compact. This choice leads to good spectral properties of the corresponding preconditioned integral equations (see Section 5). Previous investigations in acoustics would suggest that the use of the operator $P\left(N_{\alpha}\right)$, i.e

$$
\boldsymbol{\Lambda}^{\prime}=2 P\left(N_{\alpha}\right)+\alpha \mathcal{M},
$$

is sufficient to take into account all the modes. We will show that the operator $P\left(N_{\alpha}\right)$ enables the clustering of eigenvalues associated with the propagating and evanescent modes only, while the operator $\left(\mathrm{I} / 2+P\left(D_{\alpha}^{\prime}\right)\right)$ deals with the grazing modes.

To sum up, we numerically analyze three different preconditioned CFIEs:

- the Low-Order preconditioned CFIE (LO P-CFIE): integral equation (23) and the analytical preconditioner $\boldsymbol{\Lambda}_{\mathrm{LO}}^{\prime} 22$.

- the High-Order preconditioned CFIE with one term (HO(1) P-CFIE): integral equation 18) and the analytical preconditioner $\boldsymbol{\Lambda}^{\prime}(24)$ without the contribution of $P\left(D_{\alpha}^{\prime}\right), \alpha=\alpha_{H H}$.

- the High-Order preconditioned CFIE with two terms (HO(2) P-CFIE): integral equation (18) and the analytical preconditioner $\boldsymbol{\Lambda}^{\prime}(19)$ with the contribution of $P\left(D_{\alpha}^{\prime}\right), \alpha=\alpha_{H H}$.

\section{Discretization and implementation of the well-conditioned BIEs}

\subsection{Regularization and localization of the approximate adjoint DtN map}

The efficiency of the preconditioner relies on approximations of the adjoint DtN map expressed in terms of square-root operators and their inverses. Thus, it is essential to carefully compute them numerically.

Regularization. The first step consists in regularizing the square-root operator in the zone of grazing modes. Previous works in acoustics and electromagnetism have exhibited optimal damping parameters in the context of the OSRC method. A recent numerical study for elastodynamic problems [22] shows that the following parameters improve the accuracy:

$$
\varepsilon_{\gamma}=0.39 \kappa_{\gamma}^{1 / 3}\left(\mathcal{H}^{2}\right)^{1 / 3} \quad \text { with } \gamma=s, p,
$$

where $\mathcal{H}$ is the mean curvature of the boundary $\Gamma$. According to the definition of elastic waves, the values $\varepsilon_{p}$ and $\varepsilon_{s}$ are respectively the optimal choices for the Helmholtz [7] and Maxwell [43] exterior problems. We consider these two parameters in the sequel of the paper (unless indicated otherwise). 
Padé approximants. Another crucial point is to propose a robust local representation of the squareroot operators involved in (20) and (21). To this end, we use complex rational Padé approximants with a rotating branch-cut technique of angle $\theta_{1}$ 61]: for $z \in \mathbb{C}$, one has

$$
(1+z)^{1 / 2} \approx e^{i \theta_{1} / 2} R_{L_{1}}\left(e^{-i \theta_{1}}(1+z)-1\right)=C_{0}+\sum_{\ell=1}^{L_{1}} \frac{A_{\ell} z}{1+B_{\ell} z}=F_{0}-\sum_{\ell=1}^{L_{1}} \frac{A_{\ell}}{B_{\ell}\left(1+B_{\ell} z\right)},
$$

where $R_{L_{1}}$ is the standard real-valued Padé approximation of order $L_{1}$

$$
(1+z)^{1 / 2} \approx R_{L_{1}}(z)=1+\sum_{\ell=1}^{L_{1}} \frac{a_{\ell} z}{1+b_{\ell} z}
$$

with the coefficients

$$
a_{\ell}=\frac{2}{2 L+1} \sin ^{2}\left(\frac{\ell \pi}{2 L_{1}+1}\right), \quad b_{\ell}=\cos ^{2}\left(\frac{\ell \pi}{2 L_{1}+1}\right), 1 \leq \ell \leq L_{1} .
$$

The complex constants are given by

$$
\begin{gathered}
A_{\ell}=\frac{e^{-i \theta_{1} / 2} a_{\ell}}{\left(1+b_{\ell}\left(e^{-i \theta_{1}}-1\right)\right)^{2}}, \quad B_{\ell}=\frac{-e^{-i \theta_{1}} b_{\ell}}{1+b_{\ell}\left(e^{-i \theta_{1}}-1\right)}, 1 \leq \ell \leq L_{1}, \\
C_{0}=e^{i \theta_{p} / 2} R_{L_{1}}\left(e^{-i \theta_{1}}-1\right), \quad F_{0}=C_{0}+\sum_{\ell=1}^{L_{1}} \frac{A_{\ell}}{B_{\ell}} .
\end{gathered}
$$

The efficiency of these Padé complex approximants for the local representation of $(1+z)^{1 / 2}$ has been proved numerically in many previous works (e.g. [60, 7, 43]). By choosing a branch cut in the negative half-space, all the modes are modeled correctly and in particular the evanescent ones (corresponding to the region $\{z<-1 \mid \operatorname{Im}(z)=0\}$ ).

The aim is now to construct such a stable rational approximation for the function $(1+z)^{-1 / 2}$. A classical approach consists in using continued fractions [35]. Using the fixed point and residue theorems, we obtain the following rational approximation

$$
(1+z)^{-1 / 2} \approx R_{L_{2}}^{i n v}(z)=\sum_{\ell=0}^{L_{2}-1} \frac{c_{\ell}}{d_{\ell}+z}
$$

with

$$
c_{\ell}=\frac{1+\tan ^{2}\left(\frac{\pi}{2 L_{2}}\left(\frac{1}{2}+\ell\right)\right)}{L_{2}}, \quad d_{\ell}=1+\tan ^{2}\left(\frac{\pi}{2 L_{2}}\left(\frac{1}{2}+\ell\right)\right), 0 \leq \ell \leq L_{2}-1 .
$$

These real-valued coefficients correspond to a Padé approximation of the function $(1+z)^{-1 / 2}$ with branch cut along the negative real axis $\{z<-1 \mid \operatorname{Im}(z)=0\}$. A way to modify the principal determination proposed in [61] of the function is to apply a rotation of the branch cut with an angle $\theta_{2}$. We write

$$
(1+z)^{-1 / 2}=e^{i \theta_{2} / 2}\left(e^{i \theta_{2}}(1+z)\right)^{-1 / 2}=e^{i \theta_{2} / 2}\left(1+\left[e^{i \theta_{2}}(1+z)-1\right]\right)^{-1 / 2} .
$$

Using [26], we get a new approximation for the inverse square-root

$$
(1+z)^{-1 / 2} \approx e^{i \theta_{2} / 2} R_{L_{2}}^{i n v}\left(e^{i \theta_{2}}(1+z)-1\right)=\sum_{\ell=0}^{L_{2}-1} \frac{R_{\ell}}{S_{\ell}+z},
$$

where $R_{\ell}=e^{i \theta_{2} / 2} c_{\ell}$ and $S_{\ell}=1-e^{i \theta_{2}}+d_{\ell} e^{i \theta_{2}}, 0 \leq \ell \leq L_{2}-1$. 


\subsection{Discretization and optimization}

To discretize the surface $\Gamma$, we consider a triangulation with $N_{E}$ surface triangles and $N_{I}$ vertices. The polyedric interpolated surface is denoted by $\Gamma_{h}$. The discretization of the high-order adjoint DtN approximations (19) and (24) uses classical $\mathbb{P}_{1}$ boundary finite elements. We set $V_{h}=\mathbb{P}_{1}\left(\Gamma_{h}\right)$ and $\boldsymbol{V}_{h}=V_{h}^{3}$ with $\operatorname{dim} \boldsymbol{V}_{h}=N:=3 N_{I}$. The application of the preconditioner (19), namely

$$
\boldsymbol{\Lambda}^{\prime}=\left(\frac{\mathrm{I}}{2}+P\left(D_{\alpha}^{\prime}\right)\right)^{-1} P\left(N_{\alpha}\right)+\alpha \mathcal{M}
$$

is decomposed into the following three steps:

Step 1: Knowing $\boldsymbol{u} \in \boldsymbol{H}^{\frac{1}{2}}(\Gamma)$, compute the intermediate variable $\boldsymbol{v} \in \boldsymbol{H}^{\frac{1}{2}}(\Gamma)$ such that

$$
\boldsymbol{v}=P\left(N_{\alpha}\right) \boldsymbol{u} .
$$

Step 2: Solve the boundary differential equation: find $\boldsymbol{q} \in \boldsymbol{H}^{-\frac{1}{2}}(\Gamma)$ solution of

$$
\left(\frac{\mathrm{I}}{2}+P\left(D_{\alpha}^{\prime}\right)\right) \boldsymbol{q}=\boldsymbol{v}
$$

Step 3: Compute the variable $\boldsymbol{z} \in \boldsymbol{H}^{-\frac{1}{2}}(\Gamma)$

$$
\boldsymbol{z}=\boldsymbol{q}+\alpha \mathcal{M} \boldsymbol{u}
$$

In the case of the preconditioner (24), Step 2 is simply reduced to the computation of $\boldsymbol{q}=2 \boldsymbol{v}$. The variational formulation of the three steps is derived following the methodology of [22]. Nevertheless, we illustrate the derivation of the discrete weak formulation of the preconditioner with the contribution $I_{1}$ of the operator $P\left(D_{\alpha}^{\prime}\right)$ 20. As previously mentioned, the local representation of the inverse square-root operators is done with the Padé approximation (27). The damping parameters $\varepsilon_{\gamma}$ (25) are introduced to regularize square-root operators in the creeping zone. The corresponding discrete wavenumbers are expressed by $\kappa_{\gamma, \varepsilon_{h}}=\kappa_{s}+i \varepsilon_{h}$ with $\varepsilon_{h}=0.39 \kappa_{\gamma}^{1 / 3}\left(\mathcal{H}_{h}^{2}\right)^{1 / 3}$. The quantity $\mathcal{H}_{h}$ is a piecewise constant interpolation of the mean curvature $\mathcal{H}$ over $\Gamma_{h}$ on each triangle of the triangulation. The numerical evaluation of $\mathcal{H}_{h}$ comes from the relation

$$
\mathcal{H}_{h}(\boldsymbol{x})=\frac{1}{2} \operatorname{div}_{\Gamma_{h}} \boldsymbol{n}_{h}(\boldsymbol{x})=\frac{1}{2} \sum_{k=1}^{3} \sum_{j=1}^{N_{I}}\left(\boldsymbol{n}_{h}\left(\boldsymbol{a}_{j}\right) \cdot \boldsymbol{e}_{k}\right)\left(\boldsymbol{e}_{k} \cdot \nabla_{\Gamma_{h}} \varphi_{j}(\boldsymbol{x})\right), \quad \boldsymbol{x} \in \Gamma_{h},
$$

where $\boldsymbol{a}_{j}, 1 \leq j \leq N_{I}$, are the vertices of the mesh and the functions $\varphi_{j}, 1 \leq j \leq N_{I}$, the $\mathbb{P}_{1}$ basis functions on $\Gamma_{h}$. The application of the contribution $I_{1} 20$ to $\mathbf{u}_{h} \in \boldsymbol{V}_{h}$ is performed through the following steps:

(a) Solve the $L_{2}$ uncoupled equations: find $c_{h}^{\ell} \in V_{h}$ such that

$$
S_{\ell}\left(c_{h}^{\ell}, c_{h}^{\prime}\right)-\left(\kappa_{p, \varepsilon_{h}}^{-1} \nabla_{\Gamma_{h}} c_{h}^{\ell}, \kappa_{p, \varepsilon_{h}}^{-1} \nabla_{\Gamma_{h}} c_{h}^{\prime}\right)=\left(\operatorname{div}_{\Gamma_{h}} \mathrm{I}_{t} \mathbf{u}_{h}, c_{h}^{\prime}\right), c_{h}^{\prime} \in V_{h}, \ell=0, \ldots, L_{2}-1 .
$$

(b) Using step $(a)$, compute the intermediate variable $y_{h} \in V_{h}$ solution to

$$
\left(\kappa_{p, \varepsilon_{h}} y_{h}, y_{h}^{\prime}\right)=\sum_{\ell=0}^{L_{2}-1} R_{\ell}\left(c_{h}^{\ell}, y_{h}^{\prime}\right), y_{h}^{\prime} \in V_{h}
$$

(c) Independently from steps $(a)-(b)$, solve the $L_{2}$ uncoupled equations: find $w_{h}^{\ell} \in V_{h}$ such that

$$
S_{\ell}\left(w_{h}^{\ell}, w_{h}^{\prime}\right)-\left(\kappa_{s, \varepsilon_{h}}^{-1} \nabla_{\Gamma_{h}} w_{h}^{\ell}, \kappa_{s, \varepsilon_{h}}^{-1} \nabla_{\Gamma_{h}} w_{h}^{\prime}\right)=\left(\boldsymbol{n}_{h} \cdot \mathbf{u}_{h}, w_{h}^{\prime}\right), w_{h}^{\prime} \in V_{h}, \ell=0, \ldots, L_{2}-1 .
$$


(d) Using step (c), compute the intermediate variable $w_{h} \in V_{h}$ solution to

$$
\left(\kappa_{s, \varepsilon_{h}} w_{h}, w_{h}^{\prime}\right)=\sum_{\ell=0}^{L_{2}-1} R_{\ell}\left(w_{h}^{\ell}, w_{h}^{\prime}\right), \quad w_{h}^{\prime} \in V_{h} .
$$

(e) Finally compute $\boldsymbol{b}_{h} \in \boldsymbol{V}_{h}$ such that for all $\boldsymbol{b}_{h}^{\prime} \in \boldsymbol{V}_{h}$

$$
\left(\boldsymbol{b}_{h}^{\prime}, \boldsymbol{b}_{h}^{\prime}\right)=\frac{i}{2}\left(\left(y_{h}, \boldsymbol{n}_{h} \cdot \boldsymbol{b}_{h}^{\prime}\right)-\left(\nabla_{\Gamma_{h}} w_{h}, \boldsymbol{b}_{h}^{\prime}\right)\right) .
$$

These steps appear in Step 2 when the part $I_{1}$ of the operator $P\left(D_{\alpha}^{\prime}\right)$ is applied. The discretization of the term $I_{2} \sqrt{20}$ in operator $P\left(D_{\alpha}^{\prime}\right)$ (in Step 2) and of the operator $P\left(N_{\alpha}\right)$ 211) (in Step 1) is realized in the same straightforward way. We do not detail it.

Now that the discretization is introduced, the algorithm is the following. We solve the preconditioned dense non-symmetric linear system with GMRES [69] (with no restart since it is not mandatory and to have a precise idea of the impact of the preconditioning technique on the convergence). At each iteration of the solver, the solution of the preconditioned CFIE (18) requires the computation of the vector $\mathbf{Y} \in \mathbb{C}^{N}$

$$
\mathbf{Y}=\left(\frac{[\mathrm{I}]}{2}+\left[D^{\prime}\right]-\left[\boldsymbol{\Lambda}^{\prime}\right][S]\right) \mathbf{X},
$$

for any vector $\mathbf{X} \in \mathbb{C}^{N}$ given by GMRES. To speed up the solution, a FM-BEM is employed to evaluate the dense matrix-vector products involving $\left[D^{\prime}\right]$ and $[S]$ (see Section 2.3). In addition, since the preconditioners $(19)$ and $(24)$ involves only sparse matrices, the sparse direct solver SUPERLU [59, 42] is used. The Padé approximation requires to solve the sparse linear systems. Since they are uncoupled, we parallelize the solution with OpenMP. To solve each independent system we use SUPERLU that has the property to be thread-safe. For the preconditioner (19), we point out that Step 2 also requires the inversion of $\left(\mathrm{I} / 2+P\left(D_{\alpha}^{\prime}\right)\right)$. In practice, due to the use of Padé approximations, the matrix associated with this operator is never explicitly assembled and the sparse system is solved with an inner GMRES solver.

\section{Numerical efficiency of analytical preconditioners}

To study the efficiency of the different proposed preconditioned CFIEs, we consider several more or less complex geometries: a unit sphere, an ellipsoid, a cube and a sphere with cavity (see Figure 4). These scatterers are illuminated by incident plane waves of the form

$$
\boldsymbol{u}^{\mathrm{inc}}(\boldsymbol{x})=\frac{1}{\mu} e^{i \kappa_{s} \boldsymbol{x} \cdot \boldsymbol{d}}(\boldsymbol{d} \times \boldsymbol{p}) \times \boldsymbol{d}+\frac{1}{\lambda+2 \mu} e^{i \kappa_{p} \boldsymbol{x} \cdot \boldsymbol{d}}(\boldsymbol{d} \cdot \boldsymbol{p}) \boldsymbol{d}, \text { where } \boldsymbol{d} \in \mathbf{S}^{2} \text { and } \boldsymbol{p} \in \mathbb{R}^{3} .
$$

When $\boldsymbol{p}= \pm \boldsymbol{d}$, the incident plane wave oscillates along the direction of propagation (pressure wave or $\mathrm{P}$-wave). When the polarization $\boldsymbol{p}$ is orthogonal to the propagation vector $\boldsymbol{d}$, the incident plane wave oscillates in a direction orthogonal to the direction of propagation (shear wave or S-wave).

In all our simulations, the mechanical parameters are normalized and defined such that the wavenumbers satisfy $\kappa_{s}=1.5 \kappa_{p}$ (i.e. $\rho=1, \mu=1$ and $\lambda=0.1$ ). The density of points per S-wavelength $\lambda_{s}=2 \pi / \kappa_{s}$ is fixed to about $n_{\lambda_{s}}=10$, i.e. the usual criterion for high-frequency applications. The local representation of the square-root operators and their inverse, that appear in the HO P-CFIEs, is realized in the way described in Section 4.1. The parameters of Padé approximants are fixed to $\left(L_{1}, \theta_{1}\right)=\left(L_{2}, \theta_{2}\right)=(35, \pi / 3)$ (unless indicated otherwise) in Sections 5.2 and 5.3.

\subsection{Diffraction of incident plane waves by a unit sphere: numerical spectral analysis}

First, we consider the diffraction of incident plane waves by a unit sphere. A spectral decomposition, in terms of the vector spherical harmonics, of the elementary integral operators 

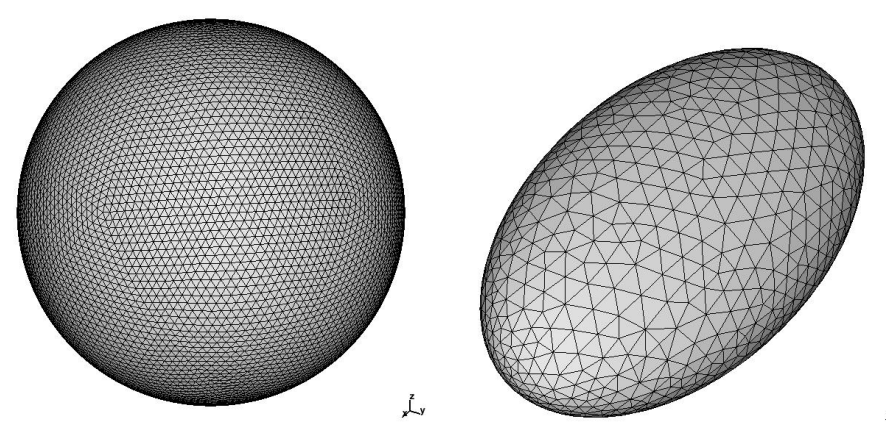

$x^{2}$
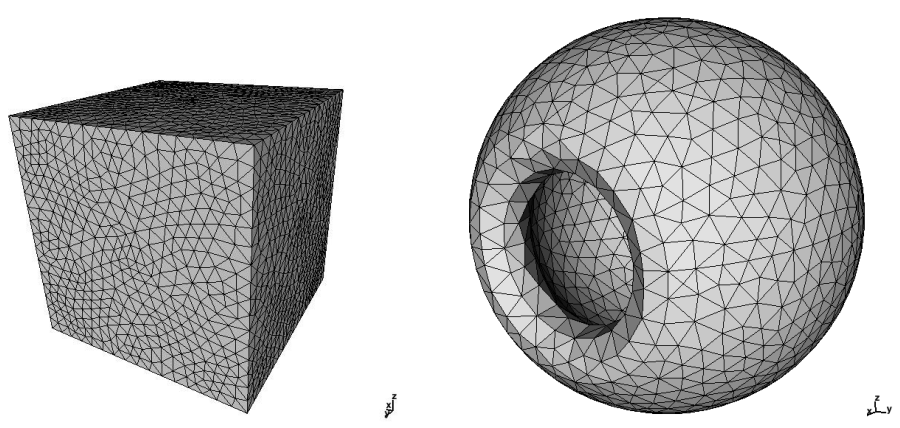

Figure 4: Illustration of the four geometries considered: a unit sphere, an ellipsoid, a cube and a sphere with cavity.

can be obtained [40. This permits to perform a detailed spectral analysis of the standard and preconditioners CFIEs with respect to both physical and discretization parameters.

In Figure 5. we report the distribution of the analytical eigenvalues of the standard CFIE (8) with $\eta=1, \kappa_{s}=16 \pi$ and $n_{\lambda_{s}}=10$. We observe a cluster of small eigenvalues close to zero. These eigenvalues correspond to grazing modes. In Figures $6 \mathrm{a}-\mathrm{b}$, we report the modulus of the smallest eigenvalue and the condition number with respect to the frequency. The modulus of the smallest eigenvalue decreases when the wavenumber $\kappa_{s}$ increases. Hence, the condition number of the CFIE deteriorates when the frequency increases. In Figure 66, we report the condition number with respect to the mesh density. We observe that it does not depend on the discretization parameter because the eigenvalues associated with evanescent modes (high-order spatial modes) are clustered.

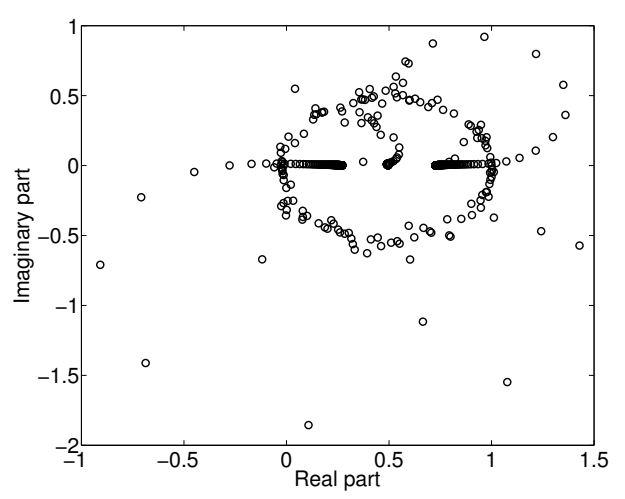

Figure 5: Distribution of the eigenvalues of the standard CFIE $\left(\eta=1, \kappa_{s}=16 \pi\right.$ and $\left.n_{\lambda_{s}}=10\right)$. 


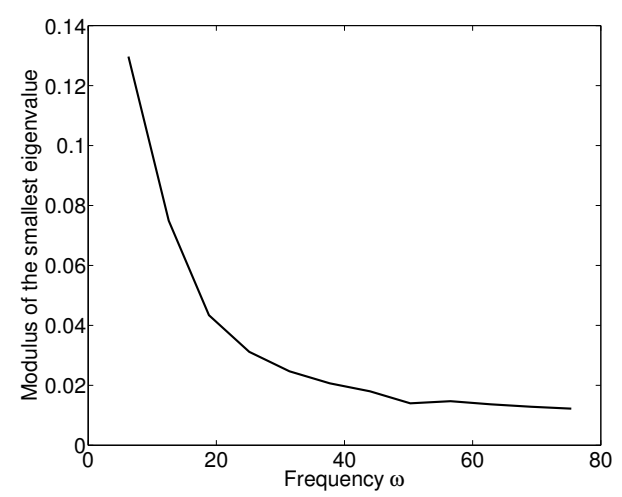

(a)

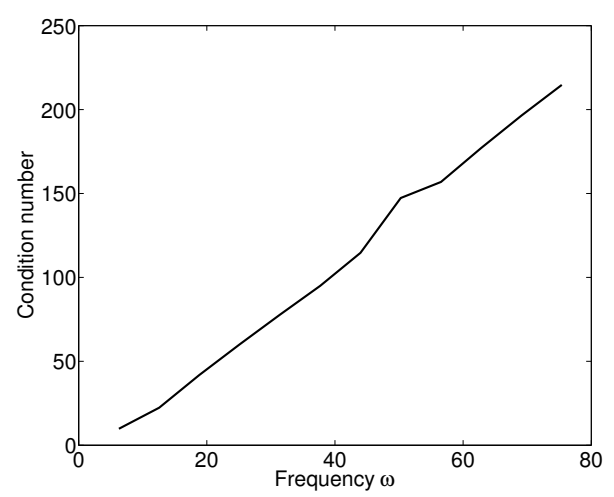

(b)

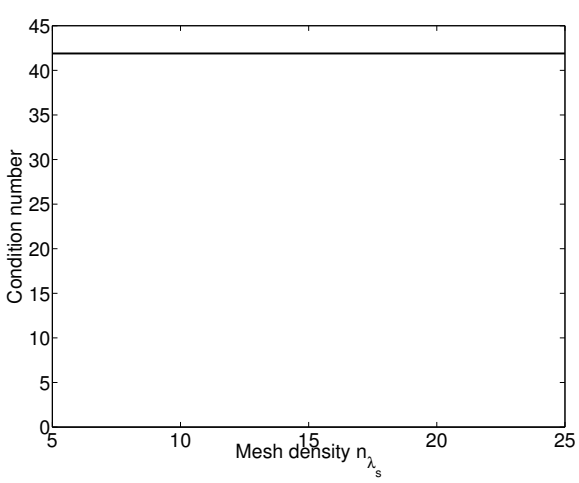

(c)

Figure 6: Study of the condition number of the standard CFIE (coupling parameter $\eta=1$ ). (a): Modulus of the smallest eigenvalue with respect to $\omega\left(n_{\lambda_{s}}=10\right)$. (b): Condition number with respect to $\omega\left(n_{\lambda_{s}}=10\right)$. (c): Condition number with respect to mesh density $n_{\lambda_{s}}\left(\kappa_{s}=6 \pi\right)$.

We compare in Figure 7 the distribution of the eigenvalues of the four CFIEs: the standard CFIE, the LO P-CFIE and the two High-Order P-CFIEs. For this spectral analysis, we consider the exact square-root operators in the approximation 190 of the adjoint DtN map. As expected, the eigenvalues of the LO P-CFIE operator are well-clustered in the hyperbolic zone only. The approximation (24) of the adjoint DtN map offers a better eigenvalue clustering than the loworder approximation. We observe an area of accumulation of the eigenvalues associated with the propagating and evanescent modes. However, these two approximations of the adjoint DtN map do not allow to shift the eigenvalues related to grazing modes around $(1,0)$ as well as the others. The best spectral configuration is obtained by considering the approximation 19 of the adjoint DtN map. Indeed, Figure 8 a confirms that taking into account the contribution relative to the double-layer potential in the preconditioner avoids the dependence of the condition number with respect to the frequency. An excellent eigenvalue clustering around the point $(1,0)$ is observed with the $\mathrm{HO}(2) \mathrm{P}-\mathrm{CFIE}$. This good property is confirmed in Figure $8 \mathrm{p}$ where the condition number remains between 1.47 and 2.25 independently of the frequency and the mesh density.

\subsection{Diffraction of incident plane waves by a unit sphere: convergence of the fast iterative solver}

For all the tests, the tolerances of the inner and outer GMRES solvers are set to $10^{-4}$ and $10^{-3}$ respectively. We consider the scattering of incident plane $\mathrm{P}$-waves with $\boldsymbol{p}=\boldsymbol{d}=(0,0,1)^{T}$, or S-waves with $\boldsymbol{d}=(0,0,1)^{T}$ and $\boldsymbol{p}=(1,0,0)^{T}$. We compare the convergence of GMRES for the iterative solution of the different standard and P-CFIEs. The number of GMRES iterations with 


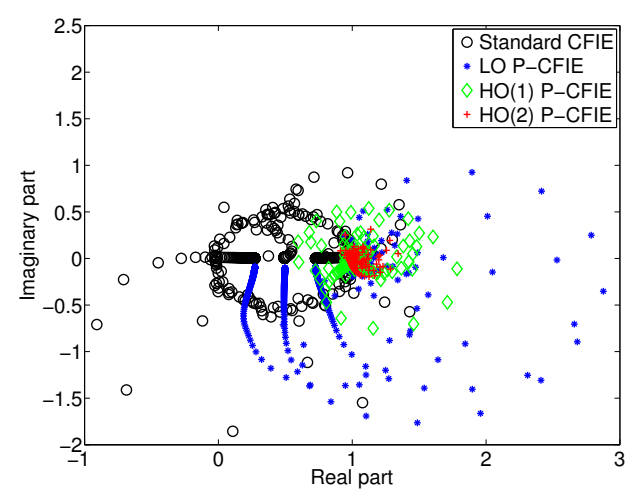

(a)

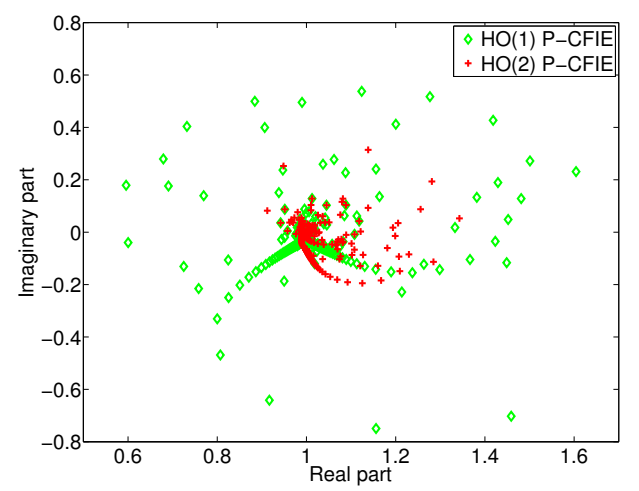

(b)

Figure 7: Distribution of the eigenvalues of the standard and different P-CFIEs $\left(\eta=1, \kappa_{s}=16 \pi\right.$ and $\left.n_{\lambda_{s}}=10\right)$.

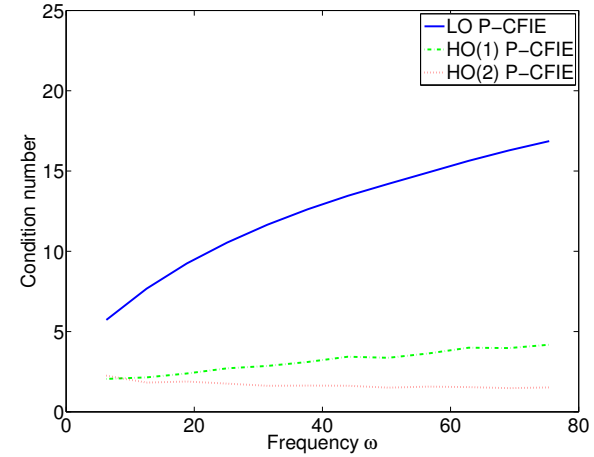

(a)

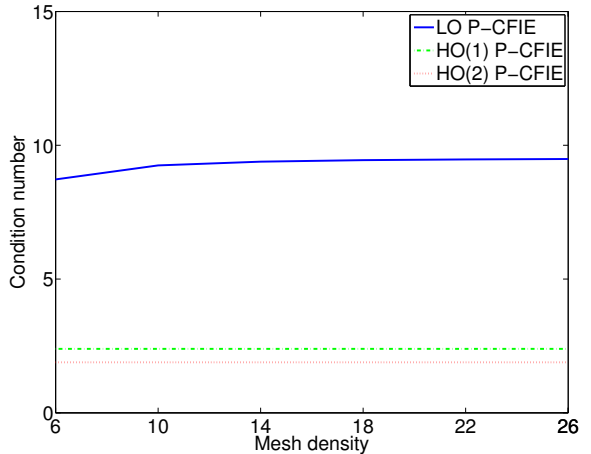

(b)

Figure 8: Study of the condition number of the P-CFIEs. (a) Condition number with respect to $\omega\left(n_{\lambda_{s}}=10\right)$. (b) Condition number with respect to the mesh density $n_{\lambda_{s}}\left(\kappa_{s}=6 \pi\right)$.

respect to the frequency $\omega$ are reported in Table11(resp. Table 22) for P-waves (resp. S-waves). In the case of the HO P-CFIEs, inner iterations are indicated in parentheses.

\begin{tabular}{cccccc}
\hline \#DOFs & $\omega$ & $\begin{array}{c}\text { \# iter } \\
\text { CFIE }\end{array}$ & $\begin{array}{c}\text { \# iter LO } \\
\text { P-CFIE }\end{array}$ & $\begin{array}{c}\text { \# iter HO(1) } \\
\text { P-CFIE }\end{array}$ & $\begin{array}{c}\text { \# iter HO(2) } \\
\text { P-CFIE }\end{array}$ \\
\hline 1926 & 4 & 18 & 8 & 7 & $5(11)$ \\
7686 & 8.25 & 27 & 8 & 6 & $4(11)$ \\
30726 & 16.5 & 51 & 9 & 6 & $3(13)$ \\
122886 & 33 & 180 & 9 & 6 & $3(13)$ \\
490629 & 66.5 & $>500$ & 9 & 6 & $3(14)$ \\
\hline
\end{tabular}

Table 1: Diffraction of P-waves by the unit sphere. Number of GMRES iterations for a fixed density of 10 points per wavelength.

These results confirm that in the case of the scattering by a unit sphere, the number of iterations of the standard CFIE drastically increases with the frequency. The LO P-CFIE is very efficient but in the case of S-waves, the number of iterations tends to increase slightly. On the other hand, the two HO P-CFIEs require a number of iterations independent of the frequency, as expected in 


\begin{tabular}{cccccc}
\hline \#DOFs & $\omega$ & $\begin{array}{c}\text { \# iter } \\
\text { CFIE }\end{array}$ & $\begin{array}{c}\text { \# iter LO } \\
\text { P-CFIE }\end{array}$ & $\begin{array}{c}\text { \# iter HO(1) } \\
\text { P-CFIE }\end{array}$ & $\begin{array}{c}\text { \# iter HO(2) } \\
\text { P-CFIE }\end{array}$ \\
\hline 1926 & 4 & 18 & 9 & 7 & $6(10)$ \\
7686 & 8.25 & 26 & 10 & 7 & $4(11)$ \\
30726 & 16.5 & 75 & 11 & 7 & $4(14)$ \\
122886 & 33 & 199 & 14 & 8 & $4(15)$ \\
490629 & 66.5 & $>500$ & 16 & 10 & $4(16)$ \\
\hline
\end{tabular}

Table 2: Diffraction of S-waves by the unit sphere. Number of GMRES iterations for a fixed density of 10 points per wavelength.

our context.

\subsection{Convergence of the fast iterative solver for non spherical scatterers}

To validate the good behavior of the HO P-CFIEs, we consider now non-spherical scatterers for which no analytical spectral analysis is available. We report again the convergence of GMRES for the diffraction of incident plane P-waves, for standard and P-CFIEs. The number of iterations with respect to the frequency $\omega$ are given in Table 3 for an ellipsoid and in Table 4 for a cube (in that case the mean curvature is set to $\sqrt{2}$ ). In the case of the HO P-CFIEs, inner iterations are indicated in parentheses.

\begin{tabular}{cccccc}
\hline \#DOFs & $\omega$ & $\begin{array}{c}\text { \# iter } \\
\text { CFIE }(\eta=1)\end{array}$ & $\begin{array}{c}\text { \# iter LO } \\
\text { P-CFIE }\end{array}$ & $\begin{array}{c}\text { \# iter HO }(1) \\
\text { P-CFIE }\end{array}$ & $\begin{array}{c}\text { \# iter HO }(2) \\
\text { P-CFIE }\end{array}$ \\
\hline 3594 & 25 & 48 & 8 & 8 & $7(12)$ \\
7644 & 30 & 122 & 10 & 8 & $7(13)$ \\
41310 & 60 & $>500$ & 11 & 9 & $8(13)$ \\
122886 & 115 & $>500$ & 13 & 12 & $10(15)$ \\
\hline
\end{tabular}

Table 3: Diffraction of P-waves by an ellipsoid. Number of GMRES iterations for a fixed density of 10 points per wavelength.

\begin{tabular}{cccccc}
\hline \#DOFs & $\omega$ & $\begin{array}{c}\text { \# iter } \\
\text { CFIE }(\eta=1)\end{array}$ & $\begin{array}{c}\text { \# iter LO } \\
\text { P-CFIE }\end{array}$ & $\begin{array}{c}\text { \# iter HO(1) } \\
\text { P-CFIE }\end{array}$ & $\begin{array}{c}\text { \# iter HO }(2) \\
\text { P-CFIE }\end{array}$ \\
\hline 1446 & 2.5 & 14 & 10 & 9 & $9(13)$ \\
6630 & 5 & 40 & 12 & 10 & $9(13)$ \\
26505 & 11 & 120 & 13 & 10 & $9(12)$ \\
105990 & 22 & $>500$ & 14 & 11 & $9(13)$ \\
\hline
\end{tabular}

Table 4: Diffraction of P-waves by a cube. Number of GMRES iterations for a fixed density of 10 points per wavelength.

For these two geometries, the number of iterations without any preconditioner drastically increases with the frequency. Even for moderate problem sizes, the number of iterations is larger than 500. The three preconditioned CFIEs are very efficient. The number of iterations are reduced to around 10 iterations for a convergence fixed to $10^{-3}$ in GMRES. Similarly to what is noted on a sphere, the high order P-CFIEs slightly reduce the number of iterations compared to the LO P-CFIE.

Finally, we consider a more complex geometry: a sphere with cavity (see Fig. 4). This trapping domain is known to be difficult due to the numerous internal reflections of waves in the cavity and the propagation of creeping waves. In Table 5 we report the number of GMRES iterations with respect to the frequency $\omega$ for two incidences of plane P-waves: $\boldsymbol{p}_{1}=\boldsymbol{d}_{1}=(-1,0,0)$ and $\boldsymbol{p}_{2}=\boldsymbol{d}_{2}=(0,0,1)$. 


\begin{tabular}{cc|cc|cc|cc}
\hline \#DOFs & $\omega$ & \multicolumn{2}{|c|}{ \# iter LO } & \multicolumn{2}{|c|}{$\begin{array}{c}\text { \# iter HO(1) } \\
\text { P-CFIE }\end{array}$} & \multicolumn{2}{|c}{ \# iter HO(2) } \\
& & \multicolumn{2}{|c|}{ P-CFIE } & \multicolumn{2}{|c|}{ PFIE } \\
\hline 11964 & 5 & 39 & 40 & 44 & 47 & $33(54)$ & $34(55)$ \\
49137 & 10 & 43 & 63 & 41 & 54 & $27(42)$ & $30(42)$ \\
98499 & 15 & 48 & 208 & 31 & 121 & $22(28)$ & $103(28)$ \\
197688 & 20 & 97 & $>500$ & 48 & 283 & $36(29)$ & $199(29)$ \\
\hline
\end{tabular}

Table 5: Diffraction of incident plane P-waves by the sphere with cavity. In each column, the first numbers give the number of GMRES iterations for $\boldsymbol{p}_{1}=\boldsymbol{d}_{1}=(-1,0,0)$ and the second numbers correspond to the incidence $\boldsymbol{p}_{2}=\boldsymbol{d}_{2}=(0,0,1)$.

In the case of sound-soft acoustic scattering problems, estimates of the condition number of the combined field integral equations have been obtained [10, 8] for different geometries of the scatterer. These studies show that the dependence of the condition number on the wavenumber is more pronounced for the case of trapping domains than for the case of a circle or a square. The same conclusion must apply in elastodynamics. The proposed preconditioner is very efficient and the GMRES iteration number for the solution of the HO(2) P-CFIE is quasi-independent on $\omega$ in the case of the sphere, the ellipsoid and the cube. The iteration number is less equal than 10 in each case. The HO(2) P-CFIE still offers an alternative to the usual CFIE when the scatterer is the sphere with cavity. As expected, the iteration number is higher than for the other scatterers but still efficiently decreased, particularly at high frequencies (36/199 against 97/ $>500$ for $\omega=20$, cf. Table 5). The iteration number is sensitive to the angle of incidence of the plane waves. The incidence $\boldsymbol{p}_{2}=\boldsymbol{d}_{2}=(0,0,1)$ normal to the cavity generates more trapping rays. Nevertheless, in the two cases considered the HO P-CFIEs are more efficient than the simple LO P-CFIE. For the second angle of incidence with $\omega=20$, the convergence is not achieved after 500 iterations for the LO P-CFIE while it converges within 200 iterations with the $\mathrm{HO}(2) \mathrm{P}-\mathrm{CFIE}$. It is worth noting that the number of inner GMRES iterations for the $\mathrm{HO}(2) \mathrm{P}$-CFIE is independent from the incidence of plane waves. To have a better understanding, we report in Figure 9 the number of iterations for the incidence $\boldsymbol{p}_{2}=\boldsymbol{d}_{2}=(0,0,1)$ for a large number of sample frequencies in the range 0-20. For all frequencies, the number of iterations is lower for the HO P-CFIEs than for the LO P-CFIE. Even if they all follow the same trend (i.e. existence of peaks at resonant frequencies of the cavity), the introduction of an accurate adjoint DtN approximation is important to keep the number of iterations reasonable at large frequencies. Close to a resonant frequency, the presence of small eigenvalues, distributed away from the cluster of eigenvalues at $(1,0)$, slows down the convergence of the iterative solver. Nevertheless, the efficiency of the HO P-CFIEs is here again highlighted.

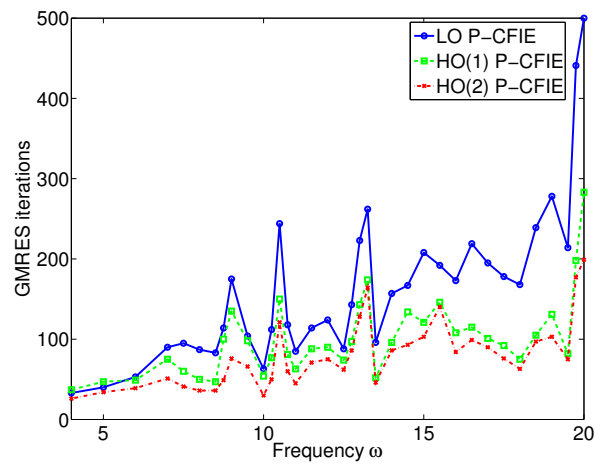

Figure 9: Diffraction of incident plane P-waves by a sphere with cavity. Number of GMRES iterations with respect to the frequency $\boldsymbol{\omega}$ for the incidence $\boldsymbol{p}_{2}=\boldsymbol{d}_{2}=(0,0,1)$. 


\section{Conclusion}

In this article, we considered the boundary integral equation method for the numerical solution of 3D high-oscillatory Dirichlet elastic exterior problems. We developed a strategy based on the use of an analytical preconditioner to accelerate the iterative solver (GMRES in our case), and the use of the Fast Multipole Method to reduce the computational cost of each iteration. We analyzed three analytic preconditioners corresponding to different approximations of the exact adjoint DtN map based on its principal pseudo-differential symbol: one approximation in the low frequency range (LO P-CFIE) and two others of higher orders (HO P-CFIEs). Numerical experiments on various geometries have shown that the convergence is strongly improved by the preconditioning with high-order approximations of the adjoint DtN map. The contribution of the double layer potential cannot be neglected in the approximation of the adjoint DtN map, especially for trapping domains.

Ongoing work concerns the derivation of such preconditioners for Neumann (or cavity) elastic exterior problems. Contrary to the acoustic and electromagnetic cases, the definition of the Neumann-to-Dirichlet preconditioner as the inverse of the DtN preconditioner is not sufficient to construct well-conditioned BIEs for Neumann scattering problems. A more extensive analysis of the DtN and Neumann-to-Dirichlet maps has to be realized in the transition region corresponding to the grazing modes.

\section{References}

[1] F. Alouges, S. Borel, And D. P. Levadoux, A stable well-conditioned integral equation for electromagnetism scattering, J. Comput. Appl. Math., 204 (2007), pp. 440-451.

[2] H. Ammari, E. Bretin, J. Garnier, H. Kang, H. Lee, and A. Wahab, Mathematical methods in elasticity imaging, Princeton Series in Applied Mathematics, Princeton University Press, Princeton, NJ, 2015.

[3] X. Antoine, H. Barucq, And A. Bendali, Bayliss-turkel-like radiation condition on surfaces of arbitrary shape, J. Math. Anal. Appl., 229 (1999), pp. 184-211.

[4] X. Antoine, A. Bendali, and M. Darbas., Analytic preconditioners for the boundary integral solution of the scattering of acoustic waves by open surfaces, Journal of Computational Acoustics, 13 (2005), pp. 477-498.

[5] X. Antoine And M. Darbas, Alternative integral equations for the iterative solution of acoustic scattering problems, Quart. J. Mech. Appl. Math., 58 (2005), pp. 107-128.

[6] X. Antoine And M. Darbas, Generalized combined field integral equations for the iterative solution of the three-dimensional Helmholtz equation, M2AN Math. Model. Numer. Anal., 41 (2007), pp. 147167.

[7] X. Antoine, M. Darbas, and Y. Y. Lu, An improved surface radiation condition for high-frequency acoustic scattering problems, Comput. Methods Appl. Mech. Engrg., 195 (2006), pp. 4060-4074.

[8] T. Beckte And E. Spence, Numerical estimation of coercivity constants for boundary integral operators in acoustic scattering, SIAM J. Numer. Anal., 49 (2011), pp. 1572-1601.

[9] J. P. Berenger, A perfectly matched layer for the absorption of electromagnetic waves, Journal of computational physics, 114 (1994), pp. 185-200.

[10] T. Betcke, S. Chandler-Wilde, I. G. Graham, S. Langdon, And M. Lindner, Condition number estimates for combined potential integral operators in acoustics and their boundary element discretization, Numerical Methods for Partial Differential Equations, 27 (2000), pp. 31-69.

[11] J. Bielak, K. Loukakis, Y. Hisada, and C. Yoshimura, Domain reduction method for threedimensional earthquake modeling in localized regions, part $i$ : Theory, Bulletin of the Seismological Society of America, 93 (2003), pp. 817-824. 
[12] M. Bonnet, Boundary integral equations methods in solids and fluids, John Wiley and sons, 1999.

[13] M. Bonnet And A. Constantinescu, Inverse problems in elasticity, Inverse Problems, 21 (2005), pp. R1-R50.

[14] Y. Boubendir AND C. TurC, Well-conditioned boundary integral equation formulations for the solution of high-frequency electromagnetic scattering problems, Computers and Mathematics with Applications, 67 (2014), pp. 1772-1805.

[15] O. Bruno, T. Elling, R. Paffenroth, and C. Turc, Electromagnetic integral equations requiring small numbers of krylov-subspace iterations, Journal of Computational Physics, 228 (2009), pp. 6169 6183.

[16] O. Bruno, T. Elling, And C. Turc, Regularized integral equations and fast high-order solvers for sound-hard acoustic scattering problems, International Journal for Numerical Methods in Engineering, 91 (2012), pp. 1045-1072.

[17] O. Bruno And S. Lintner, Second-kind integral solvers for TE and TM problems of diffraction by open arcs, Radio Science, 47 (2012).

[18] B. Carpentieri, A matrix-free two-grid preconditioner for solving boundary integral equations in electromagnetism, Computing, 77 (2006), pp. 275-296.

[19] B. Carpentieri, I. Duff, And L. Giraud, Some sparse pattern selection strategies for robust frobenius norm minimization preconditioners in electromagnetism, Numer. Linear Algebra Appl., 7 (2000), pp. 667-685.

[20] B. Carpentieri, I. S. Duff, L. Giraud, and G. Sylvand, Combining fast multipole techniques and an approximate inverse preconditioner for large parallel electromagnetics calculations, SIAM J. Sci. Comp., 27 (2005), pp. 774-792.

[21] C. Cerjan, D. Kosloff, R. Kosloff, and M. Reshef, A nonreflecting boundary condition for discrete acoustic and elastic wave equations, Geophysics, 50 (1985), pp. 705-708.

[22] S. Chaillat, M. Darbas, and F. Le Louër, Approximate local dirichlet-to-neumann map for threedimensional time-harmonic elastic waves, Computer Methods in Applied Mechanics and Engineering, 297 (2015), pp. 62-83.

[23] S. Chaillat And M. Bonnet, Recent advances on the fast multipole accelerated boundary element method for 3D time-harmonic elastodynamics, Wave Motion, 50 (2013), pp. 1090-1104.

[24] S. Chaillat, M. Bonnet, and J.-F. Semblat, A multi-level fast multipole bem for 3-d elastodynamics in the frequency domain, Computer Methods in Applied Mechanics and Engineering, 197 (2008), pp. 4233-4249.

[25] S. Chaillat, M. Bonnet, and J. F. Semblat, A multi-level fast multipole BEM for 3-D elastodynamics in the frequency domain, Comput. Methods Appl. Mech. Engng., 197 (2008), pp. 4233-4249.

[26] — - A new fast multi-domain BEM to model seismic wave propagation and amplification in 3D geological structures, Geophys. J. Int., 177 (2009), pp. 509-531.

[27] S. Challlat, J. F. Semblat, And M. Bonnet, A preconditioned 3-d multi-region fast multipole solver for seismic wave propagation in complex geometries., Commun. Comp. Phys., 11 (2012), pp. 594-609.

[28] S. Challlat, J.-F. Semblat, and M. Bonnet, A preconditioned 3-d multi-region fast multipole solver for seismic wave propagation in complex geometries, Communications in Computational Physics, 11 (2012), pp. 594-609.

[29] E. Chaluub, D. Komatitsch, J.-P. Vilotte, Y. Capdeville, B. Valette, and G. Festa, Spectral-element analysis in seismology, Advances in Geophysics, 48 (2007), pp. 365-419.

[30] S. Chandler-Wilde, I. G. Graham, S. Langdon, and E. A. Spence, Numerical-asymptotic boundary integral methods in high-frequency acoustic scattering, Acta Numerica, 21 (2012), pp. 89305. 
[31] W. C. Chew And Q. H. Liu, Perfectly matched layers for elastodynamics: a new absorbing boundary condition, Journal of Computational Acoustics, 4 (1996), pp. 341-359.

[32] S. Christiansen, Discrete fredholm properties and convergence estimates for the electric field integral equation, Math. Comp., 73 (2004), pp. 143-167.

[33] S. Christiansen and J. C. NÉdÉlec, A preconditioner for the electric field integral equation based on calderon formulas, SIAM J. Numer. Anal., 40 (2002), pp. 1100-1135.

[34] R. Clayton and B. Engquist, Absorbing boundary conditions for acoustic and elastic wave equations, Bulletin of the Seismological Society of America, 67 (1977), pp. 1529-1540.

[35] B. Cockburn and P. Joly, Maxwell equations in polarizable media, SIAM Journal on Mathematical Analysis, 19 (1988), pp. 1372-1390.

[36] C. Coifman, V. Rokhlin, and S. Wandzura, The Fast Multipole Method for the Wave Equation: A Pedestrian Prescription, IEEE Antennas and Propagation Magazine, 35 (1993), pp. 7-12.

[37] D. Colton And R. Kress, Inverse acoustic and electromagnetic scattering theory, vol. 93, Springer Science \& Business Media, 2012.

[38] M. Darbas, Generalized combined field integral equations for the iterative solution of the threedimensional Maxwell equations, Appl. Math. Lett., 19 (2006), pp. 834-839.

[39] M. Darbas, E. Darrigrand, and Y. Lafranche, Combining OSRC preconditioning and Fast Multipole Method for the Helmholtz equation, J. Comput. Phys., 236 (2013), pp. 289-316.

[40] M. Darbas And F. Le Louër, Well-conditioned boundary integral formulations for the iterative solution of elastic scattering problems, Mathematical Methods in the Applied Sciences, 38 (2015), pp. $1705-1733$.

[41] E. DARve, The fast multipole method: Numerical implementation, J. Comp. Phys., 160 (2000), pp. 195-240.

[42] J. W. Demmel, S. C. Eisenstat, J. R. Gilbert, X. S. Li, and J. W. H. Liu, A supernodal approach to sparse partial pivoting, SIAM J. Matrix Analysis and Applications, 20 (1999), pp. 720755 .

[43] M. El Bounjaji, X. Antoine, and C. Geuzaine, Approximate local magnetic-to-electric surface operators for time-harmonic maxwell's equations, Journal of Computational Physics, 279 (2014), pp. 241-260.

[44] B. Engquist And A. Majda, Radiation boundary conditions for acoustic and elastic wave calculations, Comm. Pure Appl. Math., 32 (1979), pp. 314-358.

[45] G. K. Gächter And M. J. Grote, Dirichlet-to-Neumann map for three-dimensional elastic waves, Wave Motion, 37 (2003), pp. 293-311.

[46] D. Givoli, High-order nonreflecting boundary conditions without high-order derivatives, Journal of Computational Physics, 122 (2001), pp. 849-870.

[47] R. W. Graves, Simulating seismic wave propagation in 3D elastic media using staggered-grid finite differences, Bulletin of the Seismological Society of America, 86 (1996), pp. 1091-1106.

[48] P. Hähner And G. C. Hsiao, Uniqueness theorems in inverse obstacle scattering of elastic waves, Inverse Problems, 9 (1993), pp. 525-534.

[49] L. Halpern, S. Petit-Bergez, and J. Rauch, The analysis of matched layers, Confluentes Mathematici, 3 (2011), pp. 159-236.

[50] J. S. Hesthaven and T. Warburton, Nodal discontinuous Galerkin methods: algorithms, analysis, and applications, vol. 54, Springer, 2007.

[51] G. C. Hsiao and W. L. Wendland, Boundary integral equations, vol. 164 of Applied Mathematical Sciences, Springer-Verlag, Berlin, 2008. 
[52] D. S. Jones, An approximate boundary condition in acoustics, J. Sound Vibr., 121 (1998), pp. 37-45.

[53] D. Komatitsch and J. P. Vilotte, The spectral element method: an efficient tool to simulate the seismic response of $2 D$ and $3 D$ geological structures, Bulletin of the seismological society of America, 88 (1998), pp. 368-392.

[54] G. A. Kriegsmann, A. Taflove, and K. R. Umashankar, A new formulation of electromagnetic wave scattering using the on-surface radiation condition method, IEEE Trans. Antennas Propag., 35 (1987), pp. 153-161.

[55] V. D. Kupradze, Potential methods in the theory of elasticity, Translated from the Russian by H. Gutfreund. Translation edited by I. Meroz, Israel Program for Scientific Translations, Jerusalem, 1965.

[56] V. D. Kupradze, T. G. Gegelia, M. O. Basheleřshvili, and T. V. Burchuladze, Threedimensional problems of the mathematical theory of elasticity and thermoelasticity, vol. 25 of NorthHolland Series in Applied Mathematics and Mechanics, North-Holland Publishing Co., Amsterdam, russian ed., 1979. Edited by V. D. Kupradze.

[57] D. P. Levadoux, Proposition de préconditionneurs pseudo-différentiels pour l'équation cfie de l'électromagnétisme., M2AN, 39 (2005), pp. 147-155.

[58] D. P. Levadoux And B. L. Michielsen, Nouvelles formulations intégrales pour les problèmes de diffraction d'ondes, M2AN, 38 (2004), pp. 157-175.

[59] X. Li, J. Demmel, J. Gilbert, L. Grigori, M. Shao, and I. Yamazaki, SuperLU Users' Guide, Tech. Rep. LBNL-44289, Lawrence Berkeley National Laboratory, September 1999. http: //crd.lbl.gov/ xiaoye/SuperLU/. Last update: August 2011.

[60] Y. Y. LU, A complex rational approximation of $\sqrt{1+x}$, Applied Numerical Mathematics, 27 (1998), pp. 141-154.

[61] F. A. Milinazzo, C. A. Zala, And G. H. Brooke, Rational square-root approximations for parabolic equation algorithms, J. Acoust. Soc. Am., 101 (1997), pp. 760-766.

[62] J.-C. NÉDÉLEC, Acoustic and electromagnetic equations, vol. 144 of Applied Mathematical Sciences, Springer-Verlag, New York, 2001. Integral representations for harmonic problems.

[63] S. PERNET, A well-conditioned integral equation for iterative solution of scattering problems with a variable Leontovitch boundary condition, M2AN Math. Model. Numer. Anal., 44 (2010), pp. 781-801.

[64] V. Rokhlin, Rapid solution of integral equations of scattering theory in two dimensions, Journal of Computational Physics, 86 (1990), pp. 414-439.

[65] E. H. Saenger, N. Gold, And S. A. Shapiro, Modeling the propagation of elastic waves using a modified finite-difference grid, Wave motion, 31 (2000), pp. 77-92.

[66] O. Steinbach and W. L. Wendland, The construction of some efficient preconditioners in the boundary element method, Advances in Computational Mathematics, 9 (1998), pp. 191-216.

[67] E. Van't Wout, P. Gélat, T. Betcke, and S. Arridge, A fast boundary element method for the scattering analysis of high-intensity focused ultrasound, The Journal of the Acoustical Society of America, 138 (2015), pp. 2726-2737.

[68] J. Virieux, H. Calandra, and R. E. Plessix, A review of the spectral, pseudo-spectral, finitedifference and finite-element modelling techniques for geophysical imaging, Geophysical Prospecting, 59 (2011), pp. 794-813.

[69] Y.SAAD, Iterative methods for sparse linear systems, PWS Publishing Company, Boston, 1996. 